\title{
Lifetimes of Charged and Neutral B Hadrons Using Event Topology
}

\author{
DELPHI Collaboration
}

\begin{abstract}
The lifetimes of charged and neutral $B$ hadrons have been measured using data collected by the DELPHI experiment at LEP between 1991 and 1993. B hadrons are tagged as jets with a secondary vertex and the charge of the $\mathrm{B}$ candidate is taken to be the sum of the charges of the particles in the secondary vertex. Approximately 1,434,000 multihadronic $Z^{0}$ decays yielded $1817 \mathrm{~B}$ hadron candidates. The $\mathrm{B}$ purity was estimated to be around $99.1 \pm 0.3 \%$, and $83 \%(70 \%)$ of the events measured as neutral (charged) came from neutral (charged) B's. The mean lifetimes of charged and neutral B hadrons were found to be $1.72 \pm 0.08$ (stat.) \pm 0.06 (syst.) ps and $1.58 \pm 0.11$ (stat.) \pm 0.09 (syst.) ps respectively. The ratio of their lifetimes, $\tau_{\text {charged }} / \tau_{\text {neutral }}$, was $1.09_{-0.10}^{+0.11}$ (stat.) \pm 0.08 (syst.). By making assumptions about the $\mathrm{B}_{s}^{0}$ and $\Lambda_{b}^{0}$ states, the $\mathrm{B}^{+}$and $\mathrm{B}^{0}$ meson lifetimes were determined to be $\tau_{B^{+}}=1.72 \pm 0.08$ (stat.) \pm 0.06 (syst.) ps and $\tau_{B^{0}}=1.63 \pm$ 0.14 (stat.) \pm 0.13 (syst.) ps and the ratio of their lifetimes was:

$\tau_{B^{+}} / \tau_{B^{0}}=1.06_{-0.11}^{+0.13} \pm 0.10$. The mean B lifetime was also deduced to be $\left.<\tau_{B}\right\rangle=1.64 \pm 0.06$ ( stat.) \pm 0.04 ( syst.) ps.
\end{abstract}


W.Adam ${ }^{50}$, T.Adye ${ }^{37}$, E.Agasi ${ }^{31}$, I.Ajinenko ${ }^{42}$, R.Aleksan ${ }^{39}$, G.D.Alekseev ${ }^{16}$, P.P.Allport ${ }^{22}$, S.Almehed ${ }^{24}$, S.J.Alvsvaag ${ }^{4}$, U.Amaldi $^{9}$, S.Amato $^{47}$, A.Andreazza ${ }^{28}$, M.L.Andrieux ${ }^{14}$, P.Antilogus ${ }^{25}$, V.Anykeyev ${ }^{42}$, W-D.Apel ${ }^{17}$, Y.Arnoud ${ }^{39}$, B.Asman ${ }^{44}$, J-E.Augustin ${ }^{19}$, A.Augustinus ${ }^{31}$, P.Baillon ${ }^{9}$, P.Bambade ${ }^{19}$, F.Barao $^{21}$, R.Barate $^{14}$, D.Y.Bardin ${ }^{16}$, G.J.Barker ${ }^{35}$, A.Baroncelli ${ }^{40}$, O.Barring ${ }^{24}$, J.A.Barrio ${ }^{26}$, W.Bartl ${ }^{50}$, M.J.Bates ${ }^{37}$, M.Battaglia ${ }^{15}$, M.Baubillier ${ }^{23}$, J.Baudot ${ }^{39}$, K-H.Becks ${ }^{52}$, M.Begalli ${ }^{6}$, P.Beilliere ${ }^{8}$, Yu.Belokopytov ${ }^{9}$, A.C.Benvenuti $^{5}$, M.Berggren ${ }^{41}$, D.Bertrand ${ }^{2}$, F.Bianchi ${ }^{45}$, M.Bigi ${ }^{45}$, M.S.Bilenky ${ }^{16}$, P.Billoir ${ }^{23}$, D.Bloch ${ }^{10}$, M.Blume $^{52}$, S.Blyth ${ }^{35}$, V.Bocci ${ }^{38}$, T.Bolognese ${ }^{39}$, M.Bonesini ${ }^{28}$, W.Bonivento ${ }^{28}$, P.S.L.Booth ${ }^{22}$, G.Borisov $^{42}$, C.Bosio $^{40}$, S.Bosworth ${ }^{35}$, O.Botner ${ }^{48}$, B.Bouquet ${ }^{19}$, C.Bourdarios ${ }^{9}$, T.J.V.Bowcock ${ }^{22}$, M.Bozzo ${ }^{13}$, P.Branchini $^{40}$, K.D.Brand ${ }^{36}$, R.A.Brenner ${ }^{15}$, C.Bricman ${ }^{2}$, L.Brillault ${ }^{23}$, R.C.A.Brown ${ }^{9}$, P.Bruckman ${ }^{18}$, J-M.Brunet $^{8}$, L.Bugge ${ }^{33}$, T.Buran $^{33}$, A.Buys ${ }^{9}$, M.Caccia $^{28}$, M.Calvi $^{28}$, A.J.Camacho Rozas ${ }^{41}$, T.Camporesi ${ }^{9}$, V.Canale ${ }^{38}$, M.Canepa ${ }^{13}$, K.Cankocak ${ }^{44}$, F.Cao ${ }^{2}$, F.Carena ${ }^{9}$, P.Carrilho ${ }^{47}$, L.Carroll ${ }^{22}$, C.Caso ${ }^{13}$, M.V.Castillo Gimenez ${ }^{49}$, A.Cattai ${ }^{9}$, F.R.Cavallo ${ }^{5}$, L.Cerrito ${ }^{38}$, V.Chabaud ${ }^{9}$, Ph.Charpentier ${ }^{9}$, L.Chaussard $^{25}$, J.Chauveau $^{23}$, P.Checchia ${ }^{36}$, G.A.Chelkov ${ }^{16}$, R.Chierici ${ }^{45}$, P.Chliapnikov ${ }^{42}$, P.Chochula ${ }^{7}$, V.Chorowicz ${ }^{9}$, V.Cindro ${ }^{43}$, P.Collins ${ }^{9}$, J.L.Contreras ${ }^{19}$, R.Contri ${ }^{13}$, E.Cortina ${ }^{49}$, G.Cosme ${ }^{19}$, F.Cossutti ${ }^{46}$, H.B.Crawley ${ }^{1}$, D.Crennell $^{37}$, G.Crosetti ${ }^{13}$, J.Cuevas Maestro ${ }^{34}$, S.Czellar ${ }^{15}$, E.Dahl-Jensen ${ }^{29}$, J.Dahm ${ }^{52}$, B.Dalmagne ${ }^{19}$, M.Dam $^{33}$, G.Damgaard ${ }^{29}$, A.Daum $^{17}$, P.D.Dauncey ${ }^{37}$, M.Davenport $^{9}$, W.Da Silva ${ }^{23}$, C.Defoix ${ }^{8}$, G.Della Ricca ${ }^{46}$, P.Delpierre ${ }^{27}$, N.Demaria ${ }^{35}$, A.De Angelis ${ }^{9}$, H.De Boeck ${ }^{2}$, W.De Boer ${ }^{17}$, S.De Brabandere ${ }^{2}$, C.De Clercq ${ }^{2}$, C.De La Vaissiere ${ }^{23}$, B.De Lotto ${ }^{46}$, A.De Min ${ }^{28}$, L.De Paula ${ }^{47}$, C.De Saint-Jean ${ }^{39}$, H.Dijkstra ${ }^{9}$, L.Di Ciaccio ${ }^{38}$, F.Djama ${ }^{10}$, J.Dolbeau ${ }^{8}$, M.Donszelmann ${ }^{9}$, K.Doroba ${ }^{51}$, M.Dracos ${ }^{10}$, J.Drees ${ }^{52}$, K.-A.Drees ${ }^{52}$, M.Dris $^{32}$, Y.Dufour ${ }^{8}$, F.Dupont ${ }^{14}$, D.Edsall ${ }^{1}$, R.Ehret ${ }^{17}$, G.Eigen ${ }^{4}$, T.Ekelof ${ }^{48}$, G.Ekspong ${ }^{44}$, M.Elsing ${ }^{52}$, J-P.Engel $^{10}$, N.Ershaidat ${ }^{23}$, B.Erzen ${ }^{43}$, M.Espirito Santo ${ }^{21}$, E.Falk ${ }^{24}$, D.Fassouliotis ${ }^{32}$, M.Feindt ${ }^{9}$, A.Ferrer ${ }^{49}$, T.A.Filippas ${ }^{32}$, A.Firestone ${ }^{1}$, P.-A.Fischer ${ }^{10}$, H.Foeth ${ }^{9}$, E.Fokitis ${ }^{32}$, F.Fontanelli ${ }^{13}$, F.Formenti ${ }^{9}$, B.Franek ${ }^{37}$, P.Frenkiel $^{8}$, D.C.Fries ${ }^{17}$, A.G.Frodesen ${ }^{4}$, R.Fruhwirth ${ }^{50}$, F.Fulda-Quenzer ${ }^{19}$, H.Furstenau ${ }^{9}$, J.Fuster ${ }^{49}$,

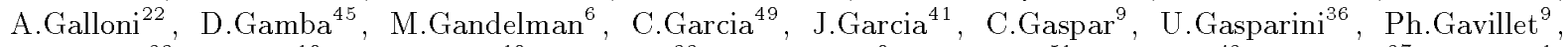
E.N.Gazis ${ }^{32}$, D.Gele ${ }^{10}$,J-P.Gerber ${ }^{10}$, M.Gibbs $^{22}$, D.Gillespie ${ }^{9}$, R.Gokieli ${ }^{51}$, B.Golob ${ }^{43}$, G.Gopal ${ }^{37}$, L.Gorn ${ }^{1}$, M.Gorski $^{51}$, Yu.Gouz ${ }^{42}$, V.Gracco ${ }^{13}$, E.Graziani ${ }^{40}$, G.Grosdidier ${ }^{19}$, P.Gunnarsson ${ }^{44}$, M.Gunther ${ }^{48}$, J.Guy ${ }^{37}$, U.Haedinger $^{17}$, F.Hahn ${ }^{52}$, M.Hahn $^{17}$, S.Hahn $^{52}$, Z.Hajduk ${ }^{18}$, A.Hallgren ${ }^{48}$, K.Hamacher ${ }^{52}$ W.Hao $^{31}$, F.J.Harris $^{35}$, V.Hedberg ${ }^{24}$, R.Henriques ${ }^{21}$, J.J.Hernandez ${ }^{49}$, P.Herquet ${ }^{2}$, H.Herr ${ }^{9}$, T.L.Hessing ${ }^{9}$, E.Higon ${ }^{49}$, H.J.Hilke ${ }^{9}$, T.S.Hill ${ }^{1}$, S-O.Holmgren ${ }^{44}$, P.J.Holt ${ }^{35}$, D.Holthuizen ${ }^{31}$, M.Houlden ${ }^{22}$, J.Hrubec ${ }^{50}$, K.Huet ${ }^{2}$, K.Hultqvist $^{44}$, P.Ioannou ${ }^{3}$, J.N.Jackson ${ }^{22}$, R.Jacobsson ${ }^{44}$, P.Jalocha ${ }^{18}$, R.Janik ${ }^{7}$, G.Jarlskog ${ }^{24}$, P.Jarry ${ }^{39}$, B.Jean-Marie ${ }^{19}$, E.K.Johansson ${ }^{44}$, L.Jonsson ${ }^{24}$, P.Jonsson ${ }^{24}$, C.Joram ${ }^{9}$, P.Juillot ${ }^{10}$, M.Kaiser ${ }^{17}$, G.Kalmus ${ }^{37}$, F.Kapusta $^{23}$, M.Karlsson ${ }^{44}$, E.Karvelas ${ }^{11}$, S.Katsanevas ${ }^{3}$, E.C.Katsoufis ${ }^{32}$, R.Keranen ${ }^{15}$, B.A.Khomenko ${ }^{16}$, N.N.Khovanski ${ }^{16}$, B.King ${ }^{22}$, N.J.Kjaer ${ }^{29}$, H.Klein ${ }^{9}$, A.Klovning ${ }^{4}$, P.Kluit ${ }^{31}$, J.H.Koehne ${ }^{17}$, B.Koene ${ }^{31}$, P.Kokkinias $^{11}$, M.Koratzinos ${ }^{9}$, K.Korcyl ${ }^{18}$, V.Kostioukhine ${ }^{42}$, C.Kourkoumelis ${ }^{3}$, O.Kouznetsov ${ }^{13}$, P.-H.Kramer ${ }^{52}$, M.Krammer ${ }^{50}$, C.Kreuter ${ }^{17}$, J.Krolikowski ${ }^{51}$, I.Kronkvist ${ }^{24}$, Z.Krumstein ${ }^{16}$, W.Krupinski ${ }^{18}{ }^{\text {, }}$ P.Kubinec $^{7}$, W.Kucewicz ${ }^{18}$, K.Kurvinen ${ }^{15}$, C.Lacasta $^{49}$, I.Laktineh ${ }^{25}$, S.Lamblot ${ }^{23}$, J.W.Lamsa ${ }^{1}$, L.Lanceri ${ }^{46}{ }^{4}$, D.W.Lane ${ }^{1}$, P.Langefeld ${ }^{52}$, V.Lapin ${ }^{42}$, I.Last ${ }^{22}$, J-P.Laugier ${ }^{39}$, R.Lauhakangas ${ }^{15}$, G.Leder ${ }^{50}$, F.Ledroit $^{14}$, V.Lefebure $^{2}$, C.K.Legan ${ }^{1}$, R.Leitner ${ }^{30}$, Y.Lemoigne ${ }^{39}$, J.Lemonne ${ }^{2}$, G.Lenzen ${ }^{52}$, V.Lepeltier ${ }^{19}$, T.Lesiak ${ }^{36}$, D.Liko $^{50}$, R.Lindner ${ }^{52}$, A.Lipniacka ${ }^{19}$, I.Lippi ${ }^{36}$, B.Loerstad ${ }^{24}$, M.Lokajicek ${ }^{12}$, J.G.Loken ${ }^{35}$, J.M.Lopez ${ }^{41}$, A.Lopez-Fernandez ${ }^{9}$, M.A.Lopez Aguera ${ }^{41}$, D.Loukas ${ }^{11}$, P.Lutz ${ }^{39}$, L.Lyons ${ }^{35}$, J.MacNaughton ${ }^{50}$, G.Maehlum $^{17}$, A.Maio $^{21}$, V.Malychev ${ }^{16}$, F.Mandl ${ }^{50}$, J.Marco ${ }^{41}$, B.Marechal ${ }^{47}$, M.Margoni ${ }^{36}$, J-C.Marin ${ }^{9}$, C.Mariotti ${ }^{40}$, A.Markou ${ }^{11}$, T.Maron ${ }^{52}$, C.Martinez-Rivero ${ }^{41}$, F.Martinez-Vidal ${ }^{49}$, S.Marti i Garcia ${ }^{49}$, F.Matorras ${ }^{41}$, C.Matteuzzi $^{28}$, G.Matthiae ${ }^{38}$, M.Mazzucato ${ }^{36}$, M.Mc Cubbin ${ }^{9}$, R.Mc Kay ${ }^{1}$, R.Mc Nulty ${ }^{22}$, J.Medbo ${ }^{48}$, C.Meroni $^{28}$, W.T.Meyer ${ }^{1}$, M.Michelotto ${ }^{36}$, E.Migliore ${ }^{45}$, L.Mirabito ${ }^{25}$, W.A.Mitaroff ${ }^{50}$, U.Mjoernmark ${ }^{24}$, T.Moa $^{44}$, R.Moeller ${ }^{29}$, K.Moenig ${ }^{9}$, M.R.Monge ${ }^{13}$, P.Morettini ${ }^{13}$, H.Mueller ${ }^{17}$, L.M.Mundim ${ }^{6}$, W.J.Murray ${ }^{37}$, B.Muryn $^{18}$, G.Myatt ${ }^{35}$, F.Naraghi ${ }^{14}$, F.L.Navarria ${ }^{5}$, S.Navas ${ }^{49}$, P.Negri ${ }^{28}$, S.Nemecek ${ }^{12}$, W.Neumann ${ }^{52}$, N.Neumeister $^{50}$, R.Nicolaidou ${ }^{3}$, B.S.Nielsen ${ }^{29}$, M.Nieuwenhuizen ${ }^{31}$, V.Nikolaenko ${ }^{10}$, P.Niss ${ }^{44}$, A.Nomerotski ${ }^{36}$, A.Normand $^{35}$, W.Oberschulte-Beckmann ${ }^{17}$, V.Obraztsov ${ }^{42}$, A.G.Olshevski ${ }^{16}$, A.Onofre ${ }^{21}$, R.Orava ${ }^{15}$, K.Osterberg ${ }^{15}$, A.Ouraou ${ }^{39}$, P.Paganini ${ }^{19}$, M.Paganoni ${ }^{28}$, P.Pages ${ }^{10}$, H.Palka ${ }^{18}$, Th.D.Papadopoulou ${ }^{32}$, L.Pape $^{9}$, C.Parkes ${ }^{35}$, F.Parodi ${ }^{13}$, A.Passeri ${ }^{40}$, M.Pegoraro ${ }^{36}$, L.Peralta ${ }^{21}$, H.Pernegger ${ }^{50}$, M.Pernicka ${ }^{50}$, A.Perrotta $^{5}$, C.Petridou ${ }^{46}$, A.Petrolini ${ }^{13}$, H.T.Phillips ${ }^{37}$, G.Piana ${ }^{13}$, F.Pierre ${ }^{39}$, M.Pimenta ${ }^{21}$, S.Plaszczynski ${ }^{19}$, O.Podobrin $^{17}$, M.E.Pol ${ }^{6}$, G.Polok ${ }^{18}$, P.Poropat ${ }^{46}$, V.Pozdniakov ${ }^{16}$, M.Prest ${ }^{46}$, P.Privitera ${ }^{38}$, N.Pukhaeva ${ }^{16}$, A.Pullia $^{28}$, D.Radojicic ${ }^{35}$, S.Ragazzi ${ }^{28}$, H.Rahmani ${ }^{32}$, J.Rames ${ }^{12}$, P.N.Ratoff ${ }^{20}$, A.L.Read ${ }^{33}$, M.Reale ${ }^{52}$, P.Rebecchi $^{19}$, N.G.Redaelli ${ }^{28}$, M.Regler ${ }^{50}$, D.Reid ${ }^{9}$, P.B.Renton ${ }^{35}$, L.K.Resvanis ${ }^{3}$, F.Richard ${ }^{19}$, J.Richardson ${ }^{22}$, J.Ridky $^{12}$, G.Rinaudo ${ }^{45}$, I.Ripp ${ }^{39}$, A.Romero ${ }^{45}$, I.Roncagliolo ${ }^{13}$, P.Ronchese ${ }^{36}$, L.Roos ${ }^{14}$, E.I.Rosenberg ${ }^{1}$, E.Rosso $^{9}$, P.Roudeau ${ }^{19}$, T.Rovelli ${ }^{5}$, W.Ruckstuhl ${ }^{31}$, V.Ruhlmann-Kleider ${ }^{39}$, A.Ruiz ${ }^{41}$, H.Saarikko ${ }^{15}$, Y.Sacquin ${ }^{39}$, A.Sadovsky ${ }^{16}$, G.Sajot ${ }^{14}$, J.Salt ${ }^{49}$, J.Sanchez ${ }^{26}$, M.Sannino ${ }^{13}$, H.Schneider ${ }^{17}$, M.A.E.Schyns ${ }^{52}$, G.Sciolla ${ }^{45}$, F.Scuri ${ }^{46}$, Y.Sedykh ${ }^{16}$, A.M.Segar ${ }^{35}$, A.Seitz ${ }^{17}$, R.Sekulin ${ }^{37}$, R.C.Shellard ${ }^{6}$, I.Siccama ${ }^{31}$, P.Siegrist $^{39}$, S.Simonetti ${ }^{39}$, F.Simonetto ${ }^{36}$, A.N.Sisakian ${ }^{16}$, B.Sitar ${ }^{7}$, T.B.Skaali ${ }^{33}$, G.Smadja $^{25}$, N.Smirnov $^{42}$, O.Smirnova ${ }^{16}$, G.R.Smith ${ }^{37}$, R.Sosnowski ${ }^{51}$, D.Souza-Santos ${ }^{6}$, T.Spassov ${ }^{21}$, E.Spiriti ${ }^{40}$, S.Squarcia $^{13}$, H.Staeck ${ }^{52}$, C.Stanescu ${ }^{40}$, S.Stapnes $^{33}$, I.Stavitski ${ }^{36}$, K.Stepaniak ${ }^{51}$, F.Stichelbaut ${ }^{9}$, A.Stocchi ${ }^{19}$, R.Strub ${ }^{10}$, B.Stugu ${ }^{4}$, M.Szczekowski ${ }^{51}$, M.Szeptycka ${ }^{51}$, T.Tabarelli ${ }^{28}$, J.P.Tavernet ${ }^{23}$, O.Tchikilev ${ }^{42}$, A.Tilquin ${ }^{27}$, J.Timmermans ${ }^{31}$, L.G.Tkatchev ${ }^{16}$, T.Todorov ${ }^{10}$, D.Z.Toet ${ }^{31}$, A.Tomaradze ${ }^{2}$, B.Tome ${ }^{21}$, L.Tortora ${ }^{40}$, 
G.Transtromer ${ }^{24}$, D.Treille ${ }^{9}$, W.Trischuk ${ }^{9}$, G.Tristram $^{8}$, A.Trombini ${ }^{19}$, C.Troncon $^{28}$, A.Tsirou ${ }^{9}$, M-L.Turluer $^{39}$, I.A.Tyapkin ${ }^{16}$, M.Tyndel ${ }^{37}$, S.Tzamarias ${ }^{22}$, B.Ueberschaer ${ }^{52}$, S.Ueberschaer ${ }^{52}$, O.Ullaland ${ }^{9}$, V.Uvarov ${ }^{42}$, G.Valenti ${ }^{5}$, E.Vallazza ${ }^{9}$, G.W.Van Apeldoorn ${ }^{31}$, P.Van Dam ${ }^{31}$, W.K.Van Doninck ${ }^{2}$, J.Van Eldik ${ }^{31}$, N.Vassilopoulos ${ }^{35}$, G.Vegni ${ }^{28}$, L.Ventura ${ }^{36}$, W.Venus ${ }^{37}$, F.Verbeure ${ }^{2}$, M.Verlato ${ }^{36}$, L.S.Vertogradov $^{16}{ }^{\text {, }}$, D.Vilanova $^{39}$, P.Vincent ${ }^{25}$, L.Vitale ${ }^{46}$, E.Vlasov ${ }^{42}$, A.S.Vodopyanov ${ }^{16}$, V.Vrba ${ }^{12}$, H.Wahlen ${ }^{52}$, C.Walck ${ }^{44}$ F.Waldner ${ }^{46}$, A.Wehr ${ }^{52}$, M.Weierstall ${ }^{52}$, P.Weilhammer ${ }^{9}$, A.M.Wetherell ${ }^{9}$, D.Wicke ${ }^{52}$, J.H.Wickens ${ }^{2}$, M.Wielers ${ }^{17}$, G.R.Wilkinson ${ }^{35}$, W.S.C.Williams ${ }^{35}$, M.Winter ${ }^{10}$, M.Witek ${ }^{9}$, K.Woschnagg ${ }^{48}$, K.Yip ${ }^{35}$, O.Yushchenko $^{42}$, F.Zach ${ }^{25}$, C.Zacharatou ${ }^{24}$, A.Zaitsev ${ }^{42}$, A.Zalewska $^{18}$, P.Zalewski ${ }^{51}$, D.Zavrtanik ${ }^{43}$, E.Zevgolatakos ${ }^{11}$, V.Zhigunov ${ }^{42}$, N.I.Zimin ${ }^{16}$, M.Zito $^{39}$, D.Zontar ${ }^{43}$, R.Zuberi ${ }^{35}$, G.C.Zucchelli ${ }^{44}$, G.Zumerle $^{36}$

\footnotetext{
${ }^{1}$ Ames Laboratory and Department of Physics, Iowa State University, Ames IA 50011, USA

${ }^{2}$ Physics Department, Univ. Instelling Antwerpen, Universiteitsplein 1, B-2610 Wilrijk, Belgium and IIHE, ULB-VUB, Pleinlaan 2, B-1050 Brussels, Belgium and Faculté des Sciences, Univ. de l'Etat Mons, Av. Maistriau 19, B-7000 Mons, Belgium ${ }^{3}$ Physics Laboratory, University of Athens, Solonos Str. 104, GR-10680 Athens, Greece ${ }^{4}$ Department of Physics, University of Bergen, Allégaten 55, N-5007 Bergen, Norway ${ }^{5}$ Dipartimento di Fisica, Università di Bologna and INFN, Via Irnerio 46, I-40126 Bologna, Italy ${ }^{6}$ Centro Brasileiro de Pesquisas Físicas, rua Xavier Sigaud 150, RJ-22290 Rio de Janeiro, Brazil and Depto. de Física, Pont. Univ. Católica, C.P. 38071 RJ-22453 Rio de Janeiro, Brazil and Inst. de Física, Univ. Estadual do Rio de Janeiro, rua São Francisco Xavier 524, Rio de Janeiro, Brazil ${ }^{7}$ Comenius University, Faculty of Mathematics and Physics, Mlynska Dolina, SK-84215 Bratislava, Slovakia ${ }^{8}$ Collège de France, Lab. de Physique Corpusculaire, IN2P3-CNRS, F-75231 Paris Cedex 05, France ${ }^{9} \mathrm{CERN}, \mathrm{CH}-1211$ Geneva 23, Switzerland

${ }^{10}$ Centre de Recherche Nucléaire, IN2P3 - CNRS/ULP - BP20, F-67037 Strasbourg Cedex, France

${ }^{11}$ Institute of Nuclear Physics, N.C.S.R. Demokritos, P.O. Box 60228, GR-15310 Athens, Greece

${ }^{12}$ FZU, Inst. of Physics of the C.A.S. High Energy Physics Division, Na Slovance 2, 180 40, Praha 8, Czech Republic

${ }^{13}$ Dipartimento di Fisica, Università di Genova and INFN, Via Dodecaneso 33, I-16146 Genova, Italy

${ }^{14}$ Institut des Sciences Nucléaires, IN2P3-CNRS, Université de Grenoble 1, F-38026 Grenoble Cedex, France

${ }^{15}$ Research Institute for High Energy Physics, SEFT, P.O. Box 9, FIN-00014 Helsinki, Finland

${ }^{16}$ Joint Institute for Nuclear Research, Dubna, Head Post Office, P.O. Box 79, 101000 Moscow, Russian Federation

${ }^{17}$ Institut für Experimentelle Kernphysik, Universität Karlsruhe, Postfach 6980, D-76128 Karlsruhe, Germany

${ }^{18}$ High Energy Physics Laboratory, Institute of Nuclear Physics, Ul. Kawiory 26a, PL-30055 Krakow 30, Poland

${ }^{19}$ Université de Paris-Sud, Lab. de l'Accélérateur Linéaire, IN2P3-CNRS, Bat 200, F-91405 Orsay Cedex, France

${ }^{20}$ School of Physics and Materials, University of Lancaster, Lancaster LA1 4YB, UK

${ }^{21}$ LIP, IST, FCUL - Av. Elias Garcia, 14-1 ${ }^{\circ}$, P-1000 Lisboa Codex, Portugal

${ }^{22}$ Department of Physics, University of Liverpool, P.O. Box 147, Liverpool L69 3BX, UK

${ }^{23}$ LPNHE, IN2P3-CNRS, Universités Paris VI et VII, Tour 33 (RdC), 4 place Jussieu, F-75252 Paris Cedex 05, France

${ }^{24}$ Department of Physics, University of Lund, Sölvegatan 14, S-22363 Lund, Sweden

${ }^{25}$ Université Claude Bernard de Lyon, IPNL, IN2P3-CNRS, F-69622 Villeurbanne Cedex, France

${ }^{26}$ Universidad Complutense, Avda. Complutense s/n, E-28040 Madrid, Spain

${ }^{27}$ Univ. d'Aix - Marseille II - CPP, IN2P3-CNRS, F-13288 Marseille Cedex 09, France

${ }^{28}$ Dipartimento di Fisica, Università di Milano and INFN, Via Celoria 16, I-20133 Milan, Italy

${ }^{29}$ Niels Bohr Institute, Blegdamsvej 17, DK-2100 Copenhagen 0, Denmark

${ }^{30}$ NC, Nuclear Centre of MFF, Charles University, Areal MFF, V Holesovickach 2, 180 00, Praha 8, Czech Republic

${ }^{31}$ NIKHEF-H, Postbus 41882, NL-1009 DB Amsterdam, The Netherlands

${ }^{32}$ National Technical University, Physics Department, Zografou Campus, GR-15773 Athens, Greece

${ }^{33}$ Physics Department, University of Oslo, Blindern, N-1000 Oslo 3, Norway

${ }^{34}$ Dpto. Fisica, Univ. Oviedo, C/P. Pérez Casas, S/N-33006 Oviedo, Spain

${ }^{35}$ Department of Physics, University of Oxford, Keble Road, Oxford OX1 3RH, UK

${ }^{36}$ Dipartimento di Fisica, Università di Padova and INFN, Via Marzolo 8, I-35131 Padua, Italy

${ }^{37}$ Rutherford Appleton Laboratory, Chilton, Didcot OX11 OQX, UK

${ }^{38}$ Dipartimento di Fisica, Università di Roma II and INFN, Tor Vergata, I-00173 Rome, Italy

${ }^{39}$ Centre d'Etude de Saclay, DSM/DAPNIA, F-91191 Gif-sur-Yvette Cedex, France

${ }^{40}$ Istituto Superiore di Sanità, Ist. Naz. di Fisica Nucl. (INFN), Viale Regina Elena 299, I-00161 Rome, Italy

${ }^{41}$ C.E.A.F.M., C.S.I.C. - Univ. Cantabria, Avda. los Castros, S/N-39006 Santander, Spain, (CICYT-AEN93-0832)

${ }^{42}$ Inst. for High Energy Physics, Serpukov P.O. Box 35, Protvino, (Moscow Region), Russian Federation

${ }^{43}$ J. Stefan Institute and Department of Physics, University of Ljubljana, Jamova 39, SI-61000 Ljubljana, Slovenia ${ }^{44}$ Fysikum, Stockholm University, Box 6730, S-113 85 Stockholm, Sweden

${ }^{45}$ Dipartimento di Fisica Sperimentale, Università di Torino and INFN, Via P. Giuria 1, I-10125 Turin, Italy

${ }^{46}$ Dipartimento di Fisica, Università di Trieste and INFN, Via A. Valerio 2, I-34127 Trieste, Italy and Istituto di Fisica, Università di Udine, I-33100 Udine, Italy

${ }^{47}$ Univ. Federal do Rio de Janeiro, C.P. 68528 Cidade Univ., Tlha do Fundão BR-21945-970 Rio de Janeiro, Brazil

${ }^{48}$ Department of Radiation Sciences, University of Uppsala, P.O. Box 535, S-751 21 Uppsala, Sweden

${ }^{49}$ IFIC, Valencia-CSIC, and D.F.A.M.N., U. de Valencia, Avda. Dr. Moliner 50, E-46100 Burjassot (Valencia), Spain

${ }^{50}$ Institut für Hochenergiephysik, Österr. Akad. d. Wissensch., Nikolsdorfergasse 18, A-1050 Vienna, Austria

${ }^{51}$ Inst. Nuclear Studies and University of Warsaw, Ul. Hoza 69, PL-00681 Warsaw, Poland

${ }^{52}$ Fachbereich Physik, University of Wuppertal, Postfach 100 127, D-42097 Wuppertal 1, Germany
} 


\section{Introduction}

This analysis is an update to the topological $\mathrm{B}^{\dagger}$ lifetimes measurement, first performed using the 1991 DELPHI data [1]. B hadron candidates are identified as distinct displaced secondary vertices of charged particle tracks which were reconstructed with high precision using the vertex detector. The charge of each $\mathrm{B}$ candidate is the total charge of the tracks assigned to the secondary vertex. The excess decay length method [2] is used to extract the lifetime.

The current analysis uses events collected from 1991 to 1993, and it therefore supplants the previous analysis. The method is dependent upon measuring the production point of charged particle tracks and relies heavily upon the silicon vertex detector.

According to the spectator model [3], the light constituent quarks are expected to play a passive role in weak decays of hadrons composed of a heavy quark and light quarks. This model predicts that the lifetimes of all $\mathrm{B}$ hadrons are equal and are determined by the lifetime of the $b$ quark. However, the lifetime differences between charmed particles clearly indicate corrections to the spectator model. These are expected [4] to give $\tau_{B^{+}} / \tau_{B^{0}}=$ $1.0+0.05 \times\left(\frac{f_{B}}{200 \mathrm{MeV}}\right)^{2}$, where $f_{B}$ is the meson decay constant, the value of which is uncertain at present.

A brief description of the detector can be found in section 2. Section 3 explains how the beam position was found. Section 4 details the event selection procedure, and the vertex reconstruction and selection is in section 5. The sample obtained is shown in section 6 , while section 7 describes the fit technique. The B lifetime results are presented in section 8 .

\section{The Apparatus}

The DELPHI detector has been described in detail elsewhere [5]. The detector was identical to that used in [1], and only a very brief summary is given here.

In the barrel region, charged particles were measured by a set of cylindrical tracking detectors whose $z$ axes are common with the electron beam and with the axis of the solenoidal magnet which produced a $1.23 \mathrm{~T}$ field. The positive $z$ axis was oriented along the electron beam direction, the radius $R$ was then defined in the plane perpendicular to $z$, and the origin of $\phi$ pointed to the centre of LEP. The $\phi$ origin was also taken as the $x$ axis, and the $y$ axis completed a right-handed coordinate system.

The Time Projection Chamber (TPC) was the main tracking device. Charged particle tracks were reconstructed in three dimensions for radii between $30 \mathrm{~cm}$ and $122 \mathrm{~cm}$ with up to 16 space points for polar angles between $39^{\circ}$ and $141^{\circ}$. Additional measurements were provided by the Inner Detector, (ID), between 12 and $28 \mathrm{~cm}$, and the Outer Detector, (OD), at around $200 \mathrm{~cm}$.

The silicon Vertex Detector (VD) [6] had three concentric layers located at average radii $6.3 \mathrm{~cm}, 8.8 \mathrm{~cm}$ and $10.9 \mathrm{~cm}$, each of which could provide one or two $R \phi$ coordinates for particles with polar angle between $43^{\circ}$ and $137^{\circ}$. The point precision in $R \phi$ was $8 \mu \mathrm{m}$.

\footnotetext{
${ }^{\dagger}$ The symbol B means charged or neutral B hadron.
} 


\section{Determination of the Interaction Region}

The position in $x$ and $y$, and the width in $x$ of the beam interaction region ${ }^{\ddagger}$ have been measured as a part of this study. This was done by forming a vertex from each hadronic event, and then combining the vertices to measure the beam position and size.

For each event a common vertex was formed from all the tracks with hits on at least two layers of the microvertex detector. The track which contributed the largest $\chi^{2}$ was removed until no track was contributing more than 10 units. Events with at least 8 tracks remaining are depleted in heavy quark species and only these were used to measure the beam intersection region. These events were accumulated in samples with a typical size of 200 events, corresponding to the cartridges written by the data acquisition system. In each sample the beam position and $x$ width were fitted from the event vertices.

The fit was a likelihood fit to the position of the vertices with two terms, one for light quark events, and the other for events which are distorted by lifetime effects. The latter component was measured by fitting the events from cartridges with more than 250 events. It was found to be reasonably described by a $16 \%$ fraction of events with a Gaussian width of $270 \mu \mathrm{m}$. (This should be compared with an initial $22 \%$ of b quarks with an approximately exponential decay length distribution of mean $3000 \mu \mathrm{m}$, and also a $c$ contribution). This fraction and width was then fixed for all cartridges, as was the $y$ size of $20 \mu \mathrm{m}$. Cartridges where less than 20 events were available to find the beamspot were excluded. The typical width in $x$ was $100-130 \mu \mathrm{m}$.

The analysis was also performed using a beamspot calculated from a single fit to all the tracks on a cartridge [7], rather than to the vertices as described here, as a cross check.

\section{Event Reconstruction}

\subsection{Hadronic Event Selection}

Only charged particles with a momentum above $0.1 \mathrm{GeV} / c$, a measured track length over $50 \mathrm{~cm}$ and an angle to the beam axis exceeding $25^{\circ}$ were used in this analysis.

The sum of the energies of these charged particles in each of the forward and backward hemispheres was required to exceed $3 \mathrm{GeV}$, and the total energy had to be more than $15 \mathrm{GeV}$. At least six charged particles were required with momenta above $0.2 \mathrm{GeV} / \mathrm{c}$. These cuts selected approximately 1,434,000 events as hadronic $Z^{0}$ decays.

The quality of the (ID) data was checked by demanding that for each event at least $65 \%$ of good barrel tracks with vertex detector hits also had ID hits. This removed $5 \%$ of events in the data.

After this hadronic event selection, the JADE jet clustering algorithm [8] was applied to the charged particles using a scaled invariant mass squared cut of 0.04 . This large value reduces the chance of splitting the $B$ decay products into two jets. For the subsequent analysis each particle was reassigned to the closest jet in $\theta-\phi$ space. In simulation this decreased the number of $B$ tracks assigned to the wrong jet from 0.03 to 0.022 . The JADE algorithm is an iterative procedure which combines tracks at each step and can never re-assign them, and it is therefore not a surprise that it is possible to improve the final track selection.

The charged particles from light hadron decays or from photon conversions can confuse an attempt to find a $\mathrm{B}$ vertex. To reduce this problem all pairs of oppositely charged

\footnotetext{
${ }^{\ddagger}$ The $y$ width was much smaller, and its uncertainty was not a significant error.
} 
particles were combined to see if they were consistent with a $\mathrm{K}_{s}^{0}$ or $\Lambda$ decay or $\gamma$ conversion. The selection criteria were designed for purity rather than efficiency, to reduce misidentification of $\mathrm{B}$ direct decay products as coming from one of these sources. About $0.17 \mathrm{~K}_{s}^{0}, 0.06 \Lambda$ and $0.48 \gamma$ per event were identified and the charged particles tagged as coming from other sources and not considered further.

After the jet finding and pair rejection, charged particles with momenta lower than $0.5 \mathrm{GeV} / c$ or a closest approach to the beam spot in the $R \phi$ plane larger than $1 \mathrm{~cm}$ were removed. Only jets with at least three remaining charged particles were accepted. All selected particles in the jet were required to have hits on at least two (of the three) layers of the vertex detector in order to be considered as reliably measured. Only one jet in five met the latter requirement. Over half the loss of jets was due to purely geometric effects coming from the finite size of the vertex detector.

The effect of these and other requirements on the combined data are listed in table 1. Also shown are the results of a full Monte Carlo simulation, starting from $\mathrm{Z}^{0}$ decays generated by the program JETSET 7.3 [9] and including particle interactions in materials and detector resolutions [10]('DELSIM'). To reproduce this resolution better, particularly in the tails of the distributions, a smearing package initially developed for B tagging was used [11]. This smeared the reconstructed track parameters using a sum of Gaussians and exponentials. The lifetimes of all the B species were set to $1.60 \mathrm{ps}$ in this simulation.

\begin{tabular}{|l|r|r|r|c|}
\hline \multirow{2}{*}{ Event Selection } & \multicolumn{2}{|c|}{ Data } & \multicolumn{2}{c|}{ Simulation } \\
\cline { 2 - 5 } & Number & Ratio & Number & Ratio \\
\hline 1) Good hadronic events & 1434425 & - & 3433432 & - \\
2) Initial jet sample & 3519393 & $2.454 \pm 0.0013$ & 8338853 & $2.429 \pm 0.001$ \\
3) Three or more particles per jet & 3096042 & $0.880 \pm 0.0002$ & 7477289 & $0.897 \pm 0.0001$ \\
4) Two or more VD hits per particle & 892571 & $0.288 \pm 0.0003$ & 2452065 & $0.328 \pm 0.0002$ \\
5) Not all from primary vertex & 407446 & $0.457 \pm 0.0007$ & 1019407 & $0.416 \pm 0.0004$ \\
6) Clear secondary vertex & 47327 & $0.116 \pm 0.0005$ & 114749 & $0.113 \pm 0.0003$ \\
\hline
\end{tabular}

Table 1: Cumulative effect of each selection on the data and the selected simulated hadronic events. The last two cuts are described in section 5

The simulation had different efficiency for selections 4) and 5) than the data. The fourth could have led to a bias in the data to low multiplicity events, but it will be seen that this was not significant. The fifth suggested that there could be slightly larger tails on either the beamspot or the track errors in the data, and its effect has been estimated at the end of section 5.1.

\subsection{Track Extrapolation Errors}

The assignment of particles to the primary vertex and secondary vertex used the extrapolated track positions in the plane perpendicular to the beam direction. The $z$ coordinate was much less precise than $R \phi$, therefore the vertex reconstruction used only the $R \phi$ projection of the charged particle tracks.

There were two contributions to the extrapolation error, $\sigma_{R \phi}$ : the intrinsic measurement resolution $\left(\sigma_{\text {meas }}\right)$, which includes alignment errors, and the multiple scattering $\left(\sigma_{\text {scat }}\right)$. For tracks with hits in at least two of the silicon layers it was parameterized by:

$$
\sigma_{R \phi}=\left[\sigma_{\text {meas }}^{2}+\frac{\sigma_{\text {scat }}^{2}}{p^{2} \sin ^{3} \theta}\right]^{\frac{1}{2}}
$$


where $p$ was the particle momentum in $\mathrm{GeV} / c$ and $\theta$ was its polar angle. Values of $\sigma_{\text {meas }}=30 \pm 3 \mu \mathrm{m}$ and $\sigma_{\text {scat }}=70 \pm 4 \mu \mathrm{m}$ were used; these represent an average over different classes of tracks, and are slightly larger than those in reference [6], for which hits in all three silicon layers were required on each charged particle.

\section{Vertex reconstruction}

Charged particles had to be assigned to the correct vertex in order to determine the charge of the $\mathrm{B}$ hadron. Each jet was examined independently. If produced by a $\mathrm{b}$ quark it will in general contain several vertices: the primary interaction, b decay, $c$ decay and perhaps s decay. However, in order to simplify the analysis, the jet was assumed to contain only the primary vertex and a single decay vertex.

A vertex was formed from all selected particles in the jet constrained to pass through the measured beam spot, for which the $x$ size was determined as described in section 3 , and the $y$ size was assumed to be $10 \mu \mathrm{m}$. A larger value of the $y$ size was used in finding the interaction region in order not to reject good vertices. The value used here was more accurate, but in practice the difference was very small. The probability of this fit in data and simulation can be seen in figure 1 . If the probability was better than $1 \%$, it was assumed that all the tracks in the jet came from the primary vertex, and such jets were not considered further.

Next, all the particles within the same jet were divided into two groups, with all possible permutations being tried. One group was used to make a vertex which was constrained by the beam spot, while a second vertex was formed using the particles in the other group with no such constraint. At least two particles were required in the secondary vertex, while any number were allowed in the primary. The probability, for which the $\chi^{2}$ was the sum of those at the primary and secondary vertices and the number of degrees of freedom was the number of particles minus two, was found for each combination.

It was required that the best combination should have a $P\left(\chi^{2}\right)$ exceeding $1 \%$. This requirement tended to reject events where the flight distance of the charmed hadron from the $B$ decay was large. It was also required that the second best combination should have a $P\left(\chi^{2}\right)$ below $1 \%$ and its $\chi^{2}$ should exceed that of the best combination by at least 4 units, i.e. $\chi_{\text {next best }}^{2}-\chi_{\text {best }}^{2}>4$. Jets which did not satisfy this requirement were regarded as ambiguous, and were rejected. The results of these vertex requirements are shown in table 1.

\subsection{Secondary Vertex Selection}

In order to select a sample of events with a high content of $B$ hadrons, a variety of selections were applied to the candidate events. These requirements are detailed in the following paragraphs.

Firstly it was required that the interaction position was well measured for the data events, and that there was no evidence of any movement within the period for which the position was averaged.

Then it was demanded that there be at least three tracks at the vertex, as simulation studies showed that B candidates making use of only two tracks had excessive background. This requirement favours charged over neutral B's as the selection efficiency was biased to low multiplicity, and the lowest multiplicity retained (three) had an odd number of tracks. $80 \%$ of the vertex candidates have only two tracks, but many of these have low mass as well. 


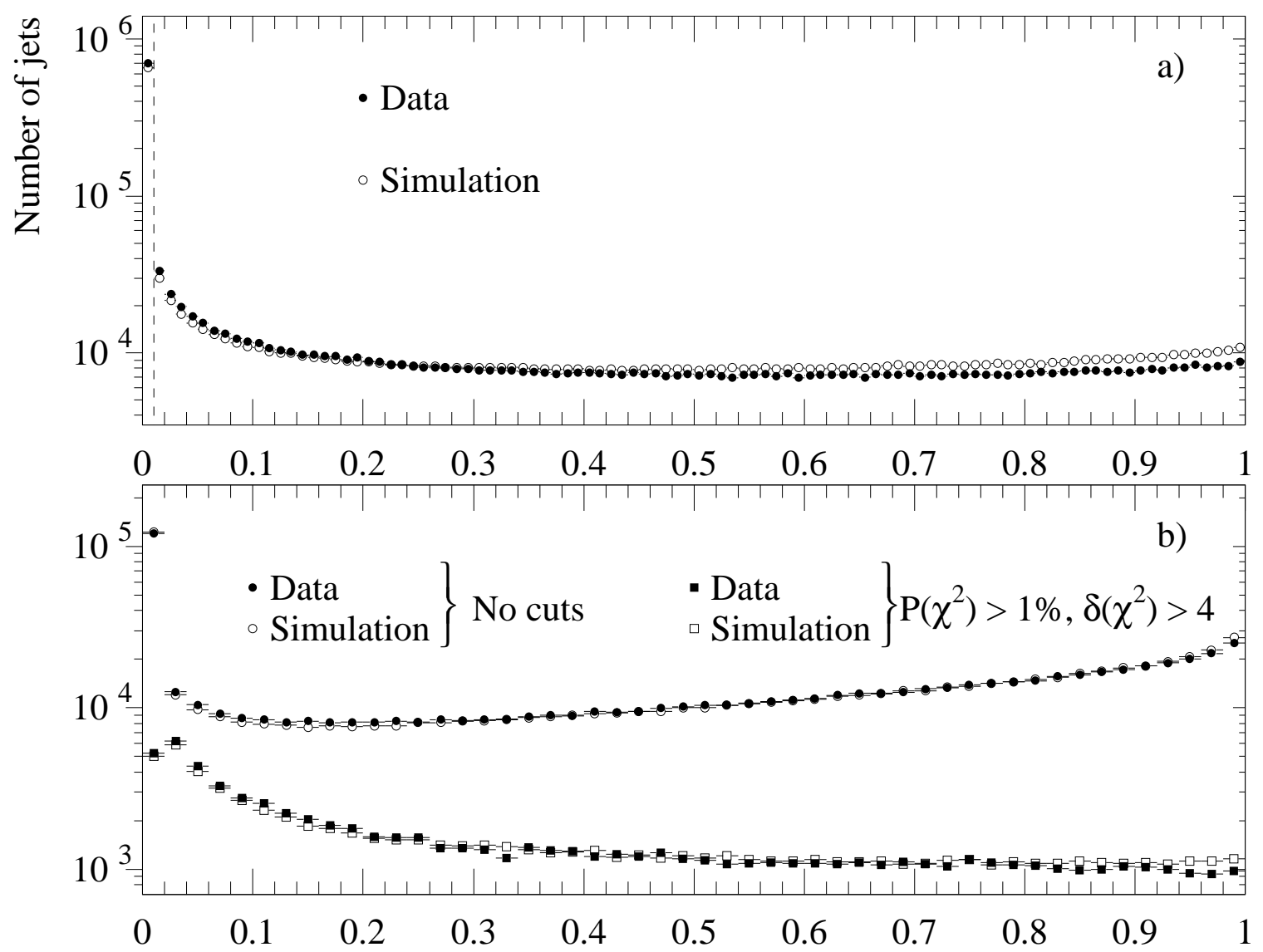

Figure 1: The upper plot shows the $P\left(\chi^{2}\right)$ distributions for one vertex formed from all the charged particle tracks and the beam spot in data and simulation. The lower plot shows the $P\left(\chi^{2}\right)$ of the best vertex as described in section 5 , both before (0) and after $(\square)$ demanding that it be distinct from the next best.

Next, the invariant mass of the candidate, $M_{v i s}$, was calculated, using only the charged particles attached to the secondary vertex and assigning them the pion mass. B decays were selected by requiring that $M_{v i s}$ exceed $2.2 \mathrm{GeV} / c^{2}$, which is above the charm kinematic limit. The mass distribution after all cuts can be seen in figure 2 a). Only a few events were found to have mass above that of the $\mathrm{B}^{0}$, which reflects the generally correct assignment of particles to vertices.

Table 2 shows the effect of this and the other selections.

Next, the vector sum of the momenta of all the particles assigned to the secondary vertex was found, and its azimuthal angle, $\phi_{\text {mom }}$, obtained. Taking the azimuthal angle, $\phi_{\text {geom }}$, of the vector joining the primary and secondary vertices, the difference $\delta \phi=$ $\phi_{\text {mom }}-\phi_{\text {geom }}$ was computed. This was expected to be near zero for B candidates because the momentum will point in the same direction as the line of flight, while background combinations coming from badly measured tracks and random combinations need not satisfy this condition.

However, $\phi_{\text {mom }}$ did not coincide with the direction of the B momentum, mostly because of the momentum carried by neutral particles; the R.M.S. difference found in simulated 

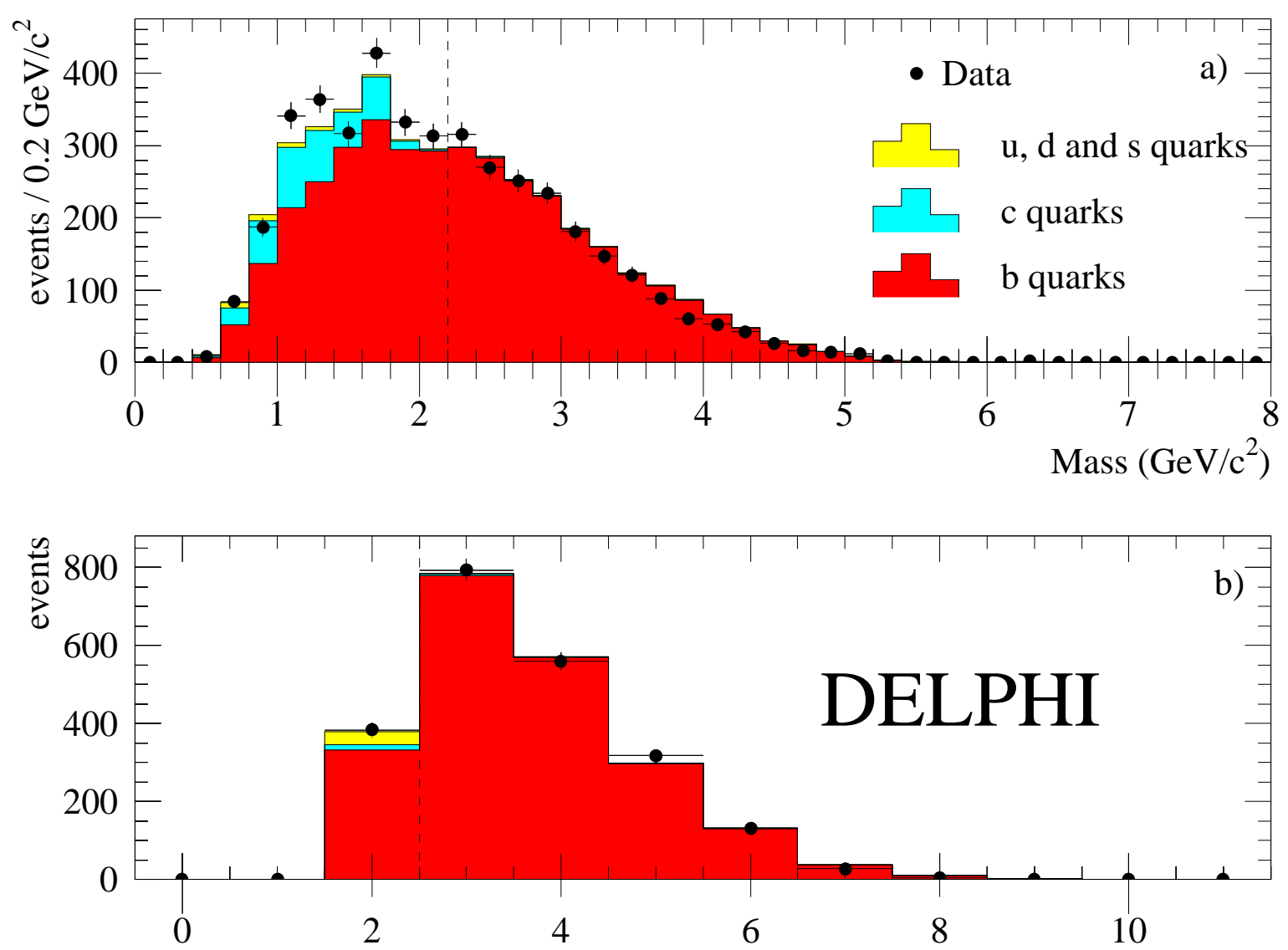

Multiplicity

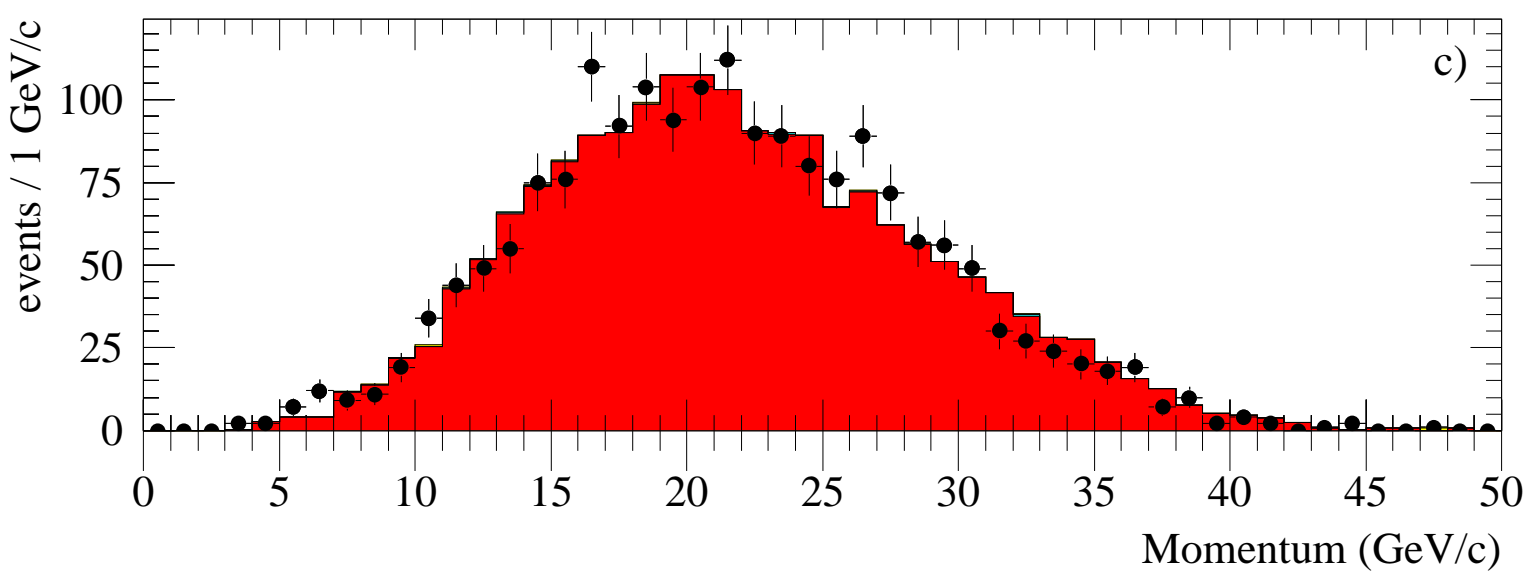

Figure 2: a) The reconstructed masses of the vertices, b) the multiplicity of the secondary vertices, and $c$ ) the summed momenta at those vertices. Only the finally accepted events are shown, except that in a) and b) those rejected only by the relevant cut are also shown. 


\begin{tabular}{|l|r|c|r|c|l|}
\hline \multirow{2}{*}{ Vertex Selection } & \multicolumn{2}{|c|}{ Data } & \multicolumn{3}{c|}{ Simulation } \\
\cline { 2 - 6 } & Number & ratio & Number & Ratio & Purity \\
\hline Initial vertex sample & 47327 & - & 114749 & - & 0.410 \\
Beam position measured & 43716 & $0.924 \pm 0.001$ & 114749 & - & 0.410 \\
Three or more tracks & 8287 & $0.190 \pm 0.011$ & 24268 & $0.211 \pm 0.006$ & 0.845 \\
Mass $>2.2 \mathrm{GeV} / c^{2}$ & 3027 & $0.365 \pm 0.018$ & 9587 & $0.395 \pm 0.010$ & 0.966 \\
$\delta \phi / \sigma_{\delta \phi}<3$ & 2767 & $0.914 \pm 0.005$ & 9111 & $0.950 \pm 0.002$ & 0.982 \\
$P\left(\chi^{2}\right)>0.10$ & 1838 & $0.664 \pm 0.011$ & 6108 & $0.682 \pm 0.006$ & 0.988 \\
$l_{R \phi}<4 \mathrm{~cm}$ & 1827 & $0.994 \pm 0.002$ & 6178 & $0.995 \pm 0.001$ & 0.992 \\
Vertex forward & 1817 & $0.995 \pm 0.002$ & 6155 & $0.996 \pm 0.001$ & 0.994 \\
\hline
\end{tabular}

Table 2: Cumulative effects of the selections applied to the vertices.

events was $50 \mathrm{mrad}$ at $M_{v i s}=2.2 \mathrm{GeV} / c^{2}$, improving to $15 \mathrm{mrad}$ at $5.0 \mathrm{GeV} / c^{2}$. Furthermore, the measurement of the primary and secondary vertices produced an error on $\delta \phi$ which decreases with increasing decay length. The error, $\sigma_{\delta \phi}$, was the quadratic sum of the error on $\phi_{m o m}$, taken from simulation and depending upon the visible mass, and the error on the position which was taken from the appropriate projection of the calculated vertex errors. Vertices were accepted if $|\delta \phi| / \sigma_{\delta \phi}$ was less than three. At this stage in the analysis, events were also retained which would have passed if their momentum had been reversed. These events are used to estimate background.

The cut on $\delta \phi$ rejects $5 \%$ of the simulated events (see table 2 ), and it would have been expected that 150 data events would not have passed. In fact, 260 were rejected, an excess of $70 \%$. The simulated events rejected were mostly light quark events in the tails of the beam spot for which the whole jet from the $Z^{0}$ was interpreted as a $B$. In the real data the beam spot had non Gaussian tails.

Events passing the above cuts were required to have a $\chi^{2}$ probability of the accepted vertex combination greater than $10 \%$, while preserving the previous criterion that no other combination should have a probability over $1 \%$. Requiring this large difference in probability reduces the chance of associating the wrong particles to the secondary vertex.

The decay length, $l_{R \phi}$, distribution for the vertices selected so far is shown in Figure 3. The decay length was required to be less than $4 \mathrm{~cm}$, because there was a small number of events found at $5.5 \mathrm{~cm}$, the radius of the beam pipe. This rejected $0.5 \%$ of events.

The negative tail of the distribution was also removed. The fraction of events affected was $0.5 \pm 0.2 \%$ in data and $0.4 \pm 0.1 \%$ in simulation. Based on these values, and the fractions of events rejected by the $\delta \phi$ cut, $(8.6 \pm 0.5 \%$ and $5.1 \pm 0.2 \%$ respectively $)$, and the background in the simulation of $0.6 \%$, the light quark contamination in the data was estimated to be between between $0.6 \%$ and $1.2 \%$. This reflected the excess events in the data seen in entry 4 ) of table 1.

The charged multiplicity distribution of the accepted secondary vertices is shown in figure $2 \mathrm{~b}$ ); the mean was $3.94 \pm 0.02$ for the data and $3.96 \pm 0.01$ for the simulation. The slightly lower probability of having two VD hits on each track in the data might have led to a lower multiplicity, but there was no firm evidence for this effect.

\section{Selected Sample Composition}

The composition of the simulated event sample before and after the selections is listed in table 3 , as is the assumed original composition of the data, which was taken from ref- 


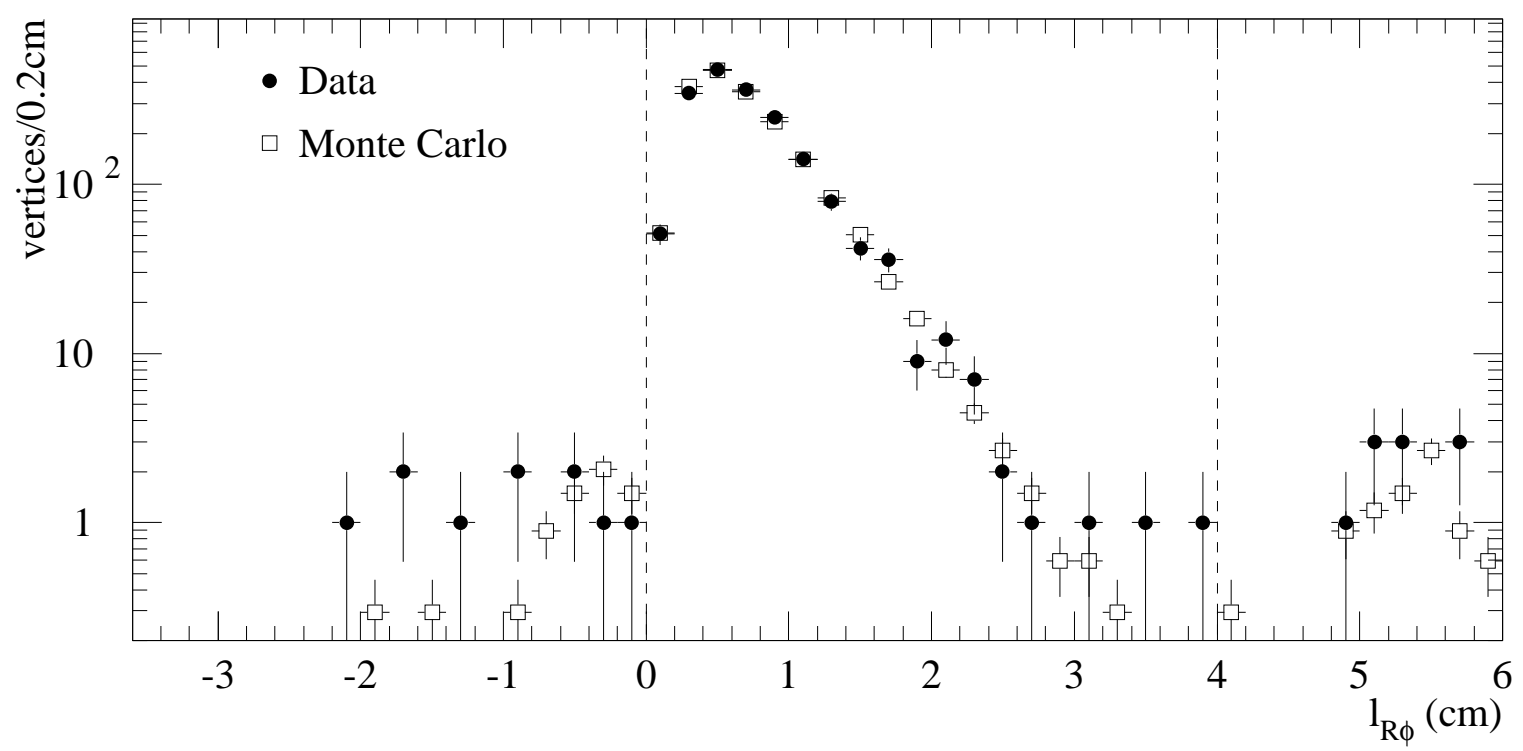

Figure 3: The $R \phi$ decay lengths of the accepted vertices in simulation and data. Negative lengths correspond to vertices which would have passed the $\delta \phi$ cut had their momentum vector been reversed. The radius of the beam pipe was $5.5 \mathrm{~cm}$

erence [12], and the estimated composition of the selected sample. The fraction of B jets after the selections was $99.4 \pm 0.1 \%$ in simulation. There was a significant enhancement in the selection of $\mathrm{B}^{+}$with respect to neutral species. This comes from the variation of efficiency with charged multiplicity, which, as discussed above, peaks at low multiplicity and favours charged $\mathrm{B}$ hadrons, and from the lower efficiency for selecting $\mathrm{D}^{+}$rather than $\mathrm{D}^{0}$ due to the difference in the lifetime. There was also a further suppression of the $\Lambda_{b}^{0}$, which was due to a lower typical value for $M_{v i s}$ in these events.

The 'other' $\mathrm{b}$ states selected in the simulation are all $\Xi_{b}$, and roughly half of these are charged. The effect of a $0.2 \%$ contribution of $\Xi_{b}^{-}$has been neglected throughout.

\begin{tabular}{|c|c|c|c|c|}
\hline \multirow{2}{*}{ Type } & \multicolumn{2}{|c|}{ Measured in simulation } & \multicolumn{2}{c|}{ Assumed in data } \\
\cline { 2 - 5 } & $\begin{array}{c}\text { Initial } \\
\text { B hadrons }\end{array}$ & $\begin{array}{c}\text { Selected } \\
\text { vertices }\end{array}$ & $\begin{array}{c}\text { Initial } \\
\text { B hadrons }\end{array}$ & $\begin{array}{c}\text { Selected } \\
\text { vertices }\end{array}$ \\
\hline $\mathrm{B}^{+}$ & $42.7 \%$ & $53.7 \pm 1.0 \%$ & $40 \%$ & $50.0 \%$ \\
$\mathrm{~B}^{0}$ & $41.9 \%$ & $34.6 \pm 0.7 \%$ & $40 \%$ & $32.9 \%$ \\
$\mathrm{~B}_{s}^{0}$ & $11.3 \%$ & $8.7 \pm 0.4 \%$ & $12 \%$ & $9.4 \%$ \\
$\Lambda_{b}^{0}$ & $3.6 \%$ & $2.0 \pm 0.2 \%$ & $8 \%$ & $6.8 \%$ \\
Other B & $0.5 \%$ & $0.4 \pm 0.1 \%$ & $0 \%$ & $0.0 \%$ \\
Background & - & $0.6 \pm 0.1 \%$ & - & $0.9 \%$ \\
\hline
\end{tabular}

Table 3: The composition of the selected event sample. In the simulation all the $\mathrm{B}$ hadron lifetimes were $1.60 \mathrm{ps}$. The DELSIM sample had a rather low rate of $\Lambda_{b}^{0}$ production, and for fits to the data the values on the right hand side of the table were assumed.

It should be noted that the selection efficiency is lifetime dependent. Table 3 shows the efficiencies if the lifetimes are identical, and the changes introduced by the lifetimes are handled in the fit described in section 7.3 . 


\section{Lifetime Fitting Procedure}

The lifetime of charged and neutral $\mathrm{B}$ hadrons was extracted from the data by means of an unbinned maximum likelihood fit to the excess decay time (see section 7.2 below). Events fall into one of four classes:

a) $B$ events where all tracks from the $B$ are correctly assigned.

b) B events where a decay track was missing from the set of tracks in the jet. This could be because of the $0.5 \mathrm{GeV} / \mathrm{c}$ momentum cut, the jet finding or the DELPHI track finding efficiency. This was assumed to be proper time independent.

c) B events where a track was misassigned from B to primary or vice versa. The $P\left(\chi^{2}\right)$ of the correct assignment of tracks must be bad for these events to be accepted, but the $P\left(\chi^{2}\right)$ of the incorrect one must be good. The probability of the correct vertex combination having a bad $P\left(\chi^{2}\right)$ was independent of proper time, so good events are removed in a manner which does not bias the lifetime of the remaining events. However, the probability of the new arrangement producing an acceptable $P\left(\chi^{2}\right)$ clearly decreases with $B$ decay length.

d) Non B events, coming from charm or lighter quarks.

All four categories are distinct, but the proper time distributions of a) and b) are similar, while the other two have their own individual distributions.

\subsection{Charge Estimation}

In order to extract the charged and neutral B lifetimes, it was important to know the accuracy of the charge estimator. The relative proportion of classes $\mathbf{a}$ ), b) and $\mathbf{c}$ ) was fitted from the data as part of the final fit, in order to minimize systematic errors. Using the quark model prediction that there are no multiply-charged B hadrons, the number of doubly and triply charged secondary vertices found was used to estimate the probability of loosing a track, or of putting a primary track into a secondary vertex.

This was done by treating each track in the $\mathrm{B}$ candidate as having an independent probability of being missed, and using binomial statistics to calculate what the observed charge distribution was for one or two tracks missed. This distribution was of course dependent upon the true charge. For simplicity, the calculation assumed that all B 's started with the same number of tracks, normally four. The sensitivity to this number was not large.

The charge estimation was binned according to how many tracks were rejected from the jet because of the low momentum or large impact parameter requirements of section 4.1. In general three quality bins were used, for 0 or 1,2 or 3 and 4 or more tracks rejected. The binomial expansions are well known, as is the probability that $m$ tracks from $n$ are missing when the mean number of missing tracks was $\mathcal{P}$. By summing over them, an expression, $P(q, Q, j)$, for the probability of observing a $\mathrm{B}$ of true charge $q$ as having measured charge $Q$ in bin $j$ was derived. In the final fit the $\mathcal{P}$ values are controlled by the observed numbers of multiply-charged events.

For the events where a track was assigned to the wrong vertex, class c), the charge distribution used was that described above, with a change of \pm 1 to account for the track which was wrong.

The charge distribution of background events was assumed to be the same in the data as in the simulation. 

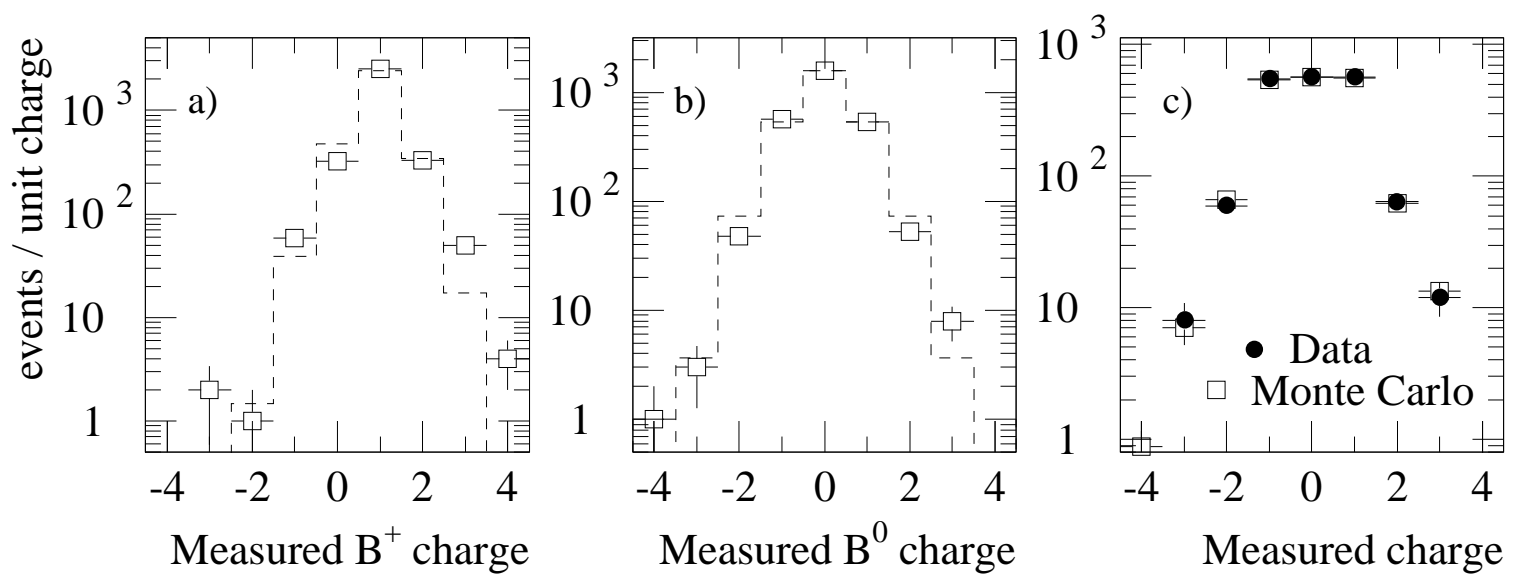

Figure 4: a) and b) show, in the simulation, the reconstructed charge for charged and neutral B hadrons respectively. Negatively charge B 's are entered in a) with their sign reversed. The line is the fitted charge distribution. c) The measured charge distribution, which combines charged and neutral.

However, there was an important correction, which came from the fact that the acceptance probability was a rapidly changing function of the number of tracks. If a $\mathrm{B}^{+}$decaying to three charged particles had one track missing it failed the selection criteria, while a $\mathrm{B}^{0}$ decaying to four prongs of which one is missed may be accepted. Because three and four prong decays dominate the selected sample, charged B mesons have their charge estimated better than neutral ones. To allow for this the values of $\mathcal{P}$ used in the fit were internally increased by $40 \%$ for $\mathrm{B}^{0}$, while for $\mathrm{B}^{+}$they were decreased by the same amount.

The procedure outlined above can be seen in figure 4 to provide a reasonable description of the simulation. The charge was measured correctly in $77 \pm 1 \%$ of the simulated charged events and $57 \pm 1 \%$ of the neutral ones, and the numbers of multiply-charged vertices, shown in Figure $4 \mathbf{b}$ ), were used to fit the parameters $\mathcal{P}_{j}$ in the data (see table 4 ).

\subsection{Excess Proper Time Estimation}

The B lifetime was fitted from the excess proper time distribution of the reconstructed decays. This requires a knowledge of the excess decay length and the B hadron velocity. The former was found from the positions of the primary and secondary vertices and by moving the secondary vertex back towards the primary along the reconstructed line of flight to find the minimum acceptable decay length of the given decay; the latter was estimated from the measured momentum and invariant mass.

\subsubsection{Excess Decay Length Measurement}

The measured decay length distribution, $l_{R \phi}$, was a convolution of the decay length of the $\mathrm{B}$ hadron with that due to the subsequent decays of $\mathrm{B}$ decay products, particularly $\mathrm{D}$ mesons. There was also an acceptance probability which was lifetime dependent. To measure the lifetime, the excess decay length, $l_{R \phi}^{e x c e s s}$, beyond the minimum required to identify the vertex, $l_{R \phi}^{\text {min }}$, was used rather than the full decay length, $l_{R \phi}$. This gives an 


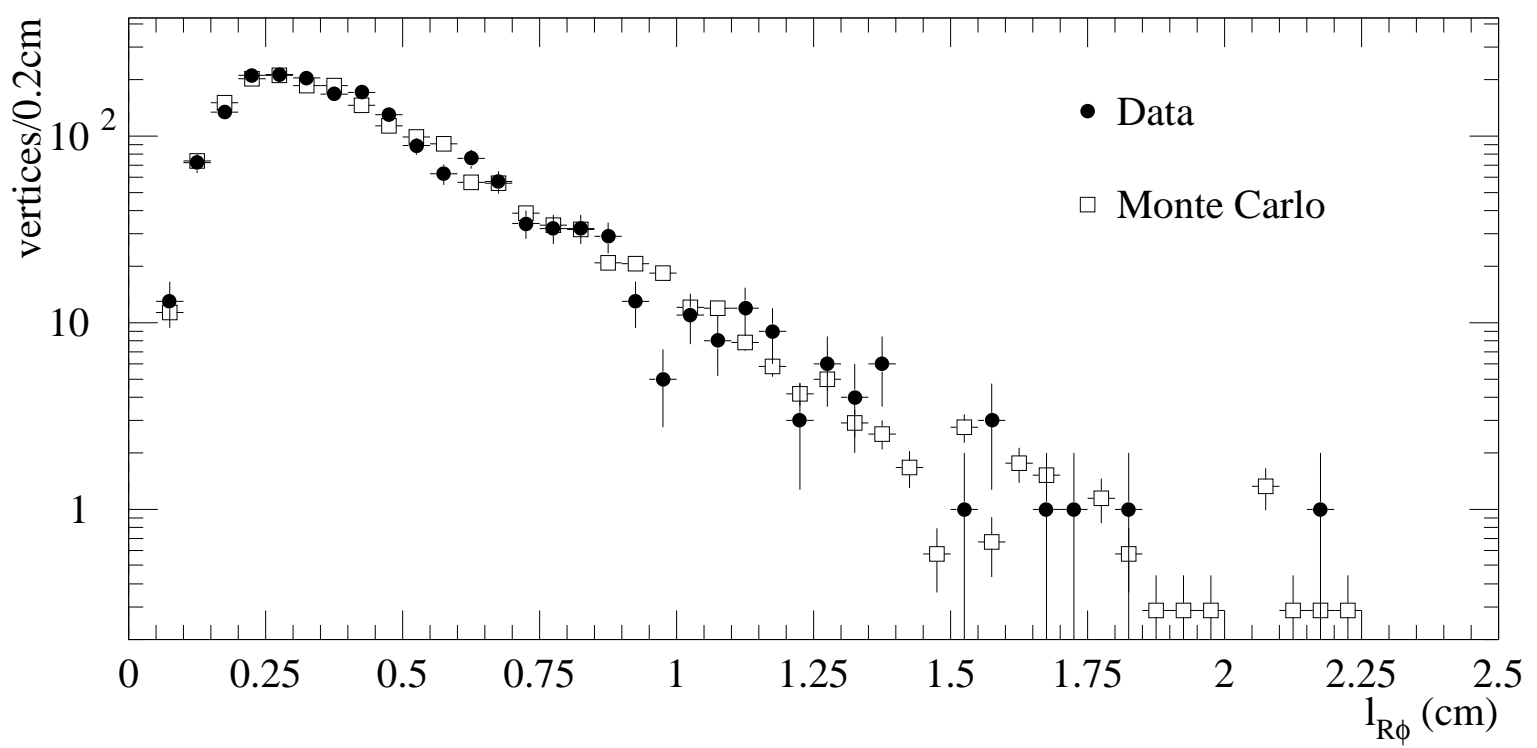

Figure 5: The minimum decay length distribution for data and simulation.

exponential distribution in lifetime and removes the dependence on the simulation of the acceptance and distortion caused by subsequent decays.

$l_{R \phi}^{\min }$ was found on an event by event basis from the data, and the measured distribution is shown in figure 5. In each accepted event, the secondary vertex and all the charged particle tracks associated to it were moved towards the primary interaction vertex. $l_{R \phi}^{\min }$ was the point at which that particular secondary vertex would just be accepted by the $\chi^{2}$ cuts described above. The time distribution of the decaying particles beyond this point was then just given by their lifetime.

The D lifetime did not distort the shape of the distribution, essentially because its effects are independent of the B flight time, and the vertices were sufficiently far from the production point that the effects near it could be neglected. The excess decay time distribution was then described by an exponential with a slope given by the B lifetime to a very good approximation ${ }^{\S}$.

The excess three dimensional decay length, $l^{\text {excess }}$, was found from this excess length in the $R \phi$ plane, $l_{R \phi}^{\text {excess }}$, as

$$
l^{\text {excess }}=\frac{l_{R \phi}^{\text {excess }}}{\sin \theta}
$$

where $\theta$ was the polar angle of the vector sum of the momenta of the charged particles assigned to the decay vertex.

\subsubsection{Boost Estimation}

The momentum of the parent $\mathrm{B}$ was found using the method of reference [13], which employs the fact that for sufficiently large boost the velocity of the B is the same as that of the observed component:

$$
\left(\frac{1}{P_{B}}\right)^{e s t}=\alpha \frac{M_{v i s}}{M_{B}} \frac{1}{P_{v i s}}
$$

$\S$ The convolution of an infinite exponential with an unknown (but finite) distribution is an exponential with the slope of the original. 
where $\left(\frac{1}{P_{B}}\right)^{e s t}$ is the estimate of the inverse of the B momentum, $M_{B}$ is the true $\mathrm{B}$ hadron mass, $M_{v i s}$ is the effective visible mass, assuming that all the particles are pions, $P_{v i s}$ is the sum of the momenta of the particles at the secondary vertex and $\alpha$ is a correction factor of order one. Substituting this into the equation for the excess proper time gives:

$$
t^{\text {excess }}=M_{B} l^{\text {excess }}\left(\frac{1}{P_{B}}\right)^{e s t}=\alpha \frac{M_{v i s} l^{\text {excess }}}{P_{v i s}}
$$

The value of $\alpha$ used was $\left(0.871+0.0331 \times M_{v i s}-0.00266 \times M_{v i s}{ }^{2}\right)^{-1}$, where $M_{v i s}$ is measured in $\mathrm{GeV} / c^{2}$. This varies from 1.08 to 1.03 in the mass range used. It deviated from one because of the exclusion of charged particles of momentum less than $0.5 \mathrm{GeV} / \mathrm{c}$, the fact that $M_{v i s}$ was calculated assuming that all the particles were pions when it was very likely that at least one was a kaon, and because of the missing transverse momentum which biased the estimator for low momentum. This parameterization and its coefficients were derived from the full simulation, and depended weakly upon the $B$ decay scheme assumed there. The largest error is due to the uncertainty in the $\mathrm{B} \rightarrow K^{+} X$ branching ratio, which contributes approximately 0.004 to the uncertainty in $\alpha$.

This estimate of the boost of the $\mathrm{B}$ had an error of $22 \%$ in the simulation, which increased the spread of the proper time distribution by a factor of $\sqrt{1.0+0.22^{2}}=1.02$. The value of $\alpha$ was compatible within statistical errors (which were less than $1 \%$ ) for $\mathrm{B}^{0}$, $\mathrm{B}^{+}$and $\mathrm{B}_{s}^{0}$, but was $10 \pm 2 \%$ larger for $\mathrm{B}$ baryons in the simulation used. This came from the assumption of the pion mass for any protons, which reduced the measured $M_{v i s}$. No correction was made for this effect; it is in any case comparable with the error on the B baryon lifetime.

The 'raw' mean momentum of the candidate B's, shown in figure $2 \mathrm{c}$ ), was $21.3 \pm 0.2 \mathrm{GeV} / \mathrm{c}$ in the data and $21.6 \pm 0.1 \mathrm{GeV} / \mathrm{c}$ in the simulation. The calculated mean momentum, using equation 3 and assuming that the mass of each $\mathrm{B}$ hadron is $5.28 \mathrm{GeV} / c^{2}$, was found to be $35.7 \pm 0.2 \mathrm{GeV} / c$ for the data and $35.6 \pm 0.1 \mathrm{GeV} / c$ for the simulation.

\subsection{The Fit Method}

A maximum likelihood fit was made to the excess proper times of the events. This included a binned estimation of the probability of reconstructing the charge wrongly, using the number of doubly and triply charged secondary vertices observed in each bin as described in section 7.1. The likelihood of event $i$ to have observed charge $Q_{i}$ and excess proper time $t_{i}^{\text {excess }}$ was taken to be:

$$
\mathcal{L}_{i}=\sum_{\nu} P\left(q_{\nu}, Q_{i}, j_{i}\right) C_{\nu} e^{-t_{i}^{e x c e s s} / \tau_{\nu}}
$$

where the sum runs over the B hadron species considered in the fit, the background, and the excess events accepted at short decay length (class c) of section 7, denoted 'confused'. The two fits considered later allow the species to be (i) the charged and neutral $\mathrm{B}$ hadrons, and (ii) the four most common species $\left(\mathrm{B}^{+}, \mathrm{B}^{0}, \mathrm{~B}_{s}^{0}, \Lambda_{b}^{0}\right)$ at LEP. $P\left(q_{\nu}, Q_{i}, j_{i}\right)$ is the probability that a $\mathrm{B}$ hadron of charge $q_{\nu}$ will be reconstructed as having charge $Q_{i}$ if the quality bin is $j_{i}, C_{\nu}$ is the normalization constant for species $\nu$, and $\tau_{\nu}$ is the mean lifetime typical of species $\nu$.

While only the B lifetime distributions are expected to be exponential, the other two contributions are both small, and an approximate description is adequate. Therefore an exponential distribution is used for each. 
$P\left(q_{\nu}, Q_{i}, i\right)$ has been described in section 7.1. It depended on the vector of parameters $\mathcal{P}_{i}$, which were allowed to vary in the fit.

The normalization constants $C_{\nu}$ are given as:

$$
C_{\nu}=\frac{F_{\nu}}{\tau_{\nu}\left[1-e^{-t^{\max } / \tau_{\nu}}\right]}
$$

where the $F_{\nu}$ are the relative fractions of the various $B$ species and the background in the selected sample, and $t^{\max }$ is the maximum allowed excess proper time of the events used in the fit and was set to 12 ps to be within the $4 \mathrm{~cm}$ decay length cut. The fractions of the selected sample, $F_{\nu}$, are related to the fractions $f_{\nu}$ which would have been observed if the lifetimes had been equal:

$$
F_{\nu}=\frac{1}{N} \times \sum_{i} \frac{f_{\nu} e^{-t_{i}^{m i n} / \tau_{\nu}}}{\sum_{\mu} f_{\mu} e^{-t_{i}^{m i n} / \tau_{\mu}}}
$$

where $N$ is the number of selected events and $t_{i}^{\text {min }}$ is the proper time of the minimum decay distance at which event $i$ would have been observed. The fractions $f_{\nu}$ are not the same as the initial fractions of the various $B$ species because of the different selection efficiencies for the different decay topologies, but are the selected fractions given in table 3 .

\section{B Lifetime Results}

\subsection{Fit to B hadron Lifetimes}

The excess proper time distributions of the charged and neutral vertices are shown in Figure 6. The fit to the average charged and neutral B lifetimes, as described below, is superimposed. In this fit it was assumed that all charged B species have one lifetime and all neutral ones have another. The relative normalization of the two species was left free to reduce the dependence upon the simulation. A background fraction of $0.9 \%$, as described in section 5.1 is included. The results of this fit are shown in table 4.

\begin{tabular}{|c|c|c|c|}
\hline Parameter & Data & \multicolumn{2}{|c|}{ Simulation } \\
\cline { 3 - 4 } & & Fit result & Correct value \\
\hline$<\tau_{\text {charged }}>(\mathrm{ps})$ & $1.72 \pm 0.08$ & $1.57 \pm 0.05$ & 1.60 \\
$\left.<\tau_{\text {neutral }}\right\rangle(\mathrm{ps})$ & $1.58 \pm 0.11$ & $1.64 \pm 0.06$ & 1.60 \\
$\tau_{\text {confused }}(\mathrm{ps})$ & $0.39_{-0.20}^{+0.44}$ & $0.63 \pm 0.17$ & \\
$f_{0}$ & $0.42 \pm 0.05$ & $0.38 \pm 0.03$ & \\
$f_{\text {confused }}$ & $0.05 \pm 0.04$ & $0.06 \pm 0.02$ & \\
$\mathcal{P}_{1}$ & $0.20 \pm 0.12$ & $0.19 \pm 0.05$ & \\
$\mathcal{P}_{2}$ & $0.32 \pm 0.10$ & $0.33 \pm 0.05$ & \\
$\mathcal{P}_{3}$ & $0.60 \pm 0.10$ & $0.63 \pm 0.06$ & \\
\hline$\left\langle\tau_{\text {charged }}\right\rangle /<\tau_{\text {neutral }}>$ & $1.09_{-0.10}^{+0.11}$ & $0.95_{-0.06}^{+0.06}$ & 1.00 \\
$\left\langle\tau_{B}\right\rangle$ & $1.64 \pm 0.06$ & $1.61 \pm 0.03$ & 1.60 \\
\hline
\end{tabular}

Table 4: Fit to average charged and neutral lifetimes, with the statistical errors shown. $\mathcal{P}$ is defined in section 7.1, and $f_{0}$ is the fraction of neutral $\mathrm{B}$ hadrons which would have been selected if the lifetimes had been equal. The last two entries are not independent parameters, but the errors have been calculated separately.

The three classes of $\mathcal{P}$ contain 380, 580 and 857 events respectively. The charge measurement is poor in $\mathcal{P}_{3}$; but it should be remembered that even events with no 

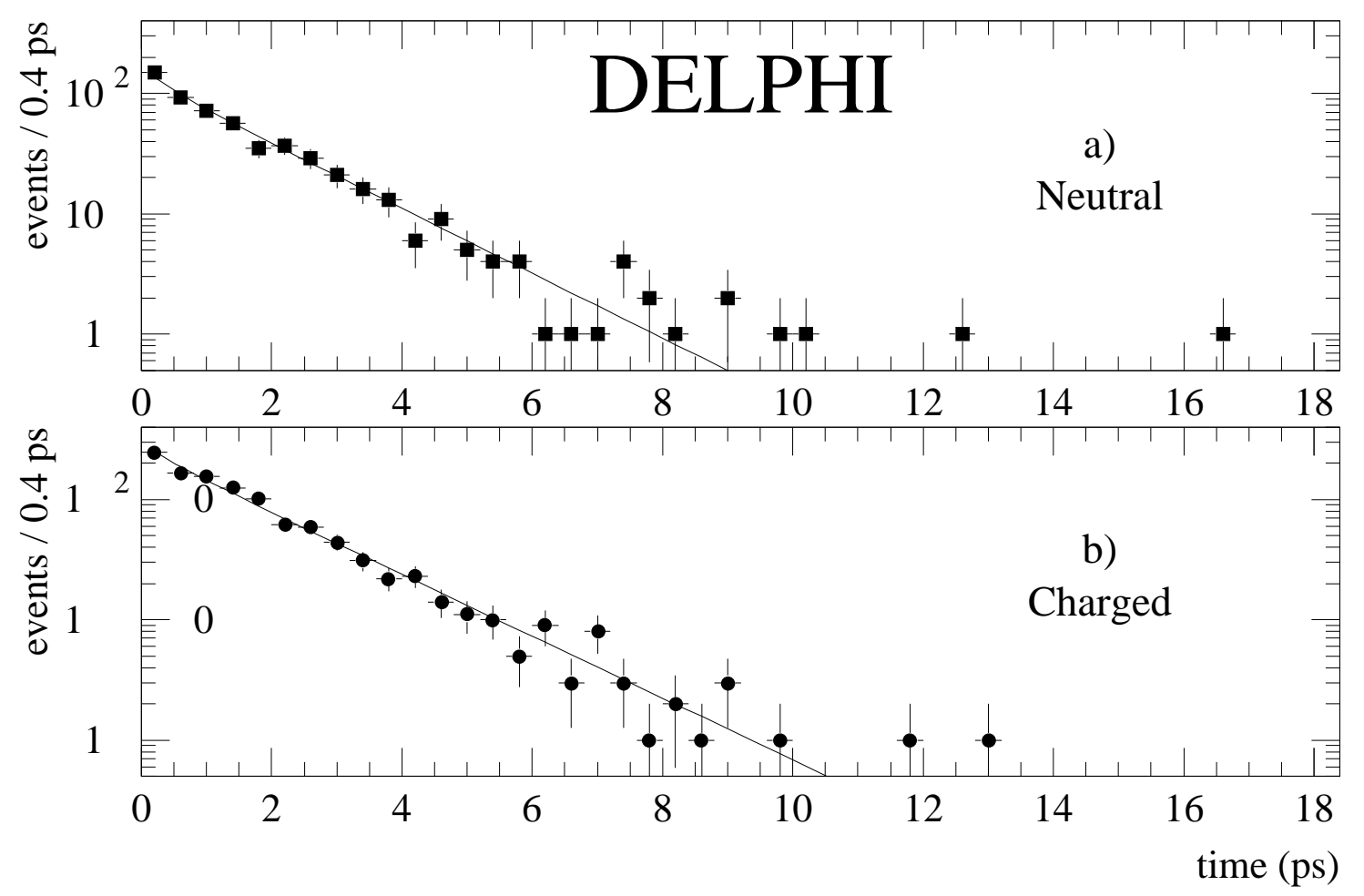

Figure 6: The excess proper time distributions of the charged and neutral vertices, with the fit result superimposed. The slight curvature is because the fit is a sum of exponentials.

charge information whatsoever would still contribute to measuring the mean lifetime. The advantage of splitting the charge error into three bins is that the relatively precise measurements are used to their full.

The lifetime ratio and $\left\langle\tau_{B}\right\rangle$ in table 4 are the results of a fit in which the charged and neutral $B$ lifetimes were replaced with the ratio of charged to neutral lifetimes, and the mean $\mathrm{B}$ lifetime at production in LEP, assuming that the composition is as given in table 3 and that all the neutral species have the same lifetime. This number is shown for comparison with the more careful average performed below in section 8.2.

The systematic errors estimated in the analysis are discussed below and summarized in table 5:

\begin{tabular}{|l|l|c|c|c|c|}
\hline & Effect Investigated & $\left\langle\tau_{\text {charged }}\right\rangle$ & $\left\langle\tau_{\text {neutral }}\right\rangle$ & $\frac{\left\langle\tau_{\text {char }}\right\rangle}{\left\langle\tau_{\text {nut }}\right\rangle}$ & $\left\langle\tau_{B}\right\rangle$ \\
\hline 1$)$ & Background fraction/lifetime & $\pm 0.01 \mathrm{ps}$ & $\pm 0.02 \mathrm{ps}$ & \pm 0.02 & $\pm 0.01 \mathrm{ps}$ \\
$2)$ & Background charge & $\pm 0.01 \mathrm{ps}$ & $\pm 0.01 \mathrm{ps}$ & \pm 0.01 & $\pm 0.00 \mathrm{ps}$ \\
$3)$ & Binning of charge & $\pm 0.03 \mathrm{ps}$ & $\pm 0.01 \mathrm{ps}$ & \pm 0.03 & $\pm 0.00 \mathrm{ps}$ \\
$4)$ & Relative charge width & $\pm 0.01 \mathrm{ps}$ & $\pm 0.01 \mathrm{ps}$ & \pm 0.01 & $\pm 0.00 \mathrm{ps}$ \\
$5)$ & Momentum estimation & $\pm 0.01 \mathrm{ps}$ & $\pm 0.03 \mathrm{ps}$ & \pm 0.02 & $\pm 0.02 \mathrm{ps}$ \\
$6)$ & Maximum time in fit & $\pm 0.01 \mathrm{ps}$ & $\pm 0.05 \mathrm{ps}$ & \pm 0.04 & $\pm 0.02 \mathrm{ps}$ \\
$7)$ & Possible analysis bias & $\pm 0.05 \mathrm{ps}$ & $\pm 0.06 \mathrm{ps}$ & \pm 0.05 & $\pm 0.03 \mathrm{ps}$ \\
\hline & Total & $\pm 0.06 \mathrm{ps}$ & $\pm 0.09 \mathrm{ps}$ & \pm 0.08 & $\pm 0.04 \mathrm{ps}$ \\
\hline
\end{tabular}

Table 5: Systematic uncertainties in the fit to the average charged and neutral B lifetimes. 
1. The background fraction was varied from $0.6 \%$ to $1.2 \%$ and its lifetime halved and doubled to estimate a systematic error.

2. The charge distribution used for the background has also been changed to double the number of events with charge greater than 1 . This resulted in a very small systematic error.

3. The charge error was normally binned by the number of tracks deliberately excluded. For comparison this was changed to depend upon the measured mass, to which it was not as sensitive. The observed change in the results has been taken as a systematic error.

4. The value of $\mathcal{P}$ was altered inside the fit by $-40 \%(+40 \%)$ for truly charged(neutral) B's. This was changed to $-20 \%(+20 \%)$ and $-60 \%(+60 \%)$, which give the ratio of the error as 1.5 and 4 respectively, and while the fit results for some of the technical parameters changed, the lifetimes were almost unaffected. The change produced in the fitted function was much larger than the discrepancies between the fit and the simulated events seen in figures $4 \mathbf{a}$ and $4 \mathbf{b}$.

5. The conversion from decay length to boost was another potential cause of systematic error. From the difference between the value of $\alpha$ applicable for the $\Lambda_{b}^{0}$ and the other neutral $\mathrm{B}$ species, and the uncertainty of the fraction of $\Lambda_{b}^{0}$ in the sample, a $2 \%$ or 0.03 ps systematic error was deduced. For the charged $B$ the expression for $\alpha$ was expected to be good to $0.5 \%$.

6. The variation of lifetime seen in figure 7 as a function of the maximum proper time in the fit was taken as a systematic error for the neutral B lifetime. That seen in the charged $B$ was not statistically significant. The results are quoted for a maximum time allowed of $12 \mathrm{ps}$.
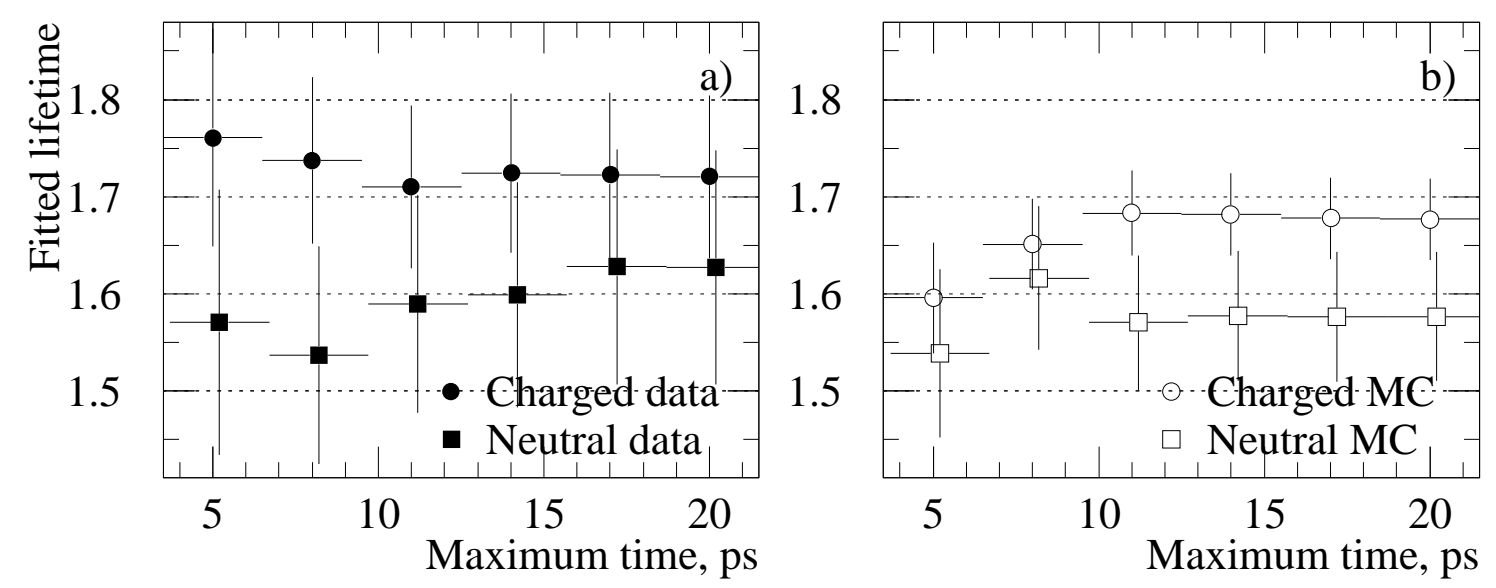

Figure 7: The variation of the results with maximum time. Data is shown in a) and simulation, with all lifetimes $1.6 \mathrm{ps}$, in b). The neutral results have been offset slightly to the right for clarity.

7. The statistical error of the simulation was taken as a further systematic error, as there could be unforseen effects of that magnitude. For example, the classifications introduced in section 7 are not perfectly valid, but overall the fit reproduces the simulated input. 
Variation of the exact values of the cuts used produced changes in the results compatible with the statistical fluctuations expected (e.g. $1.68 \pm 0.07$ and $1.62 \pm 0.10 \mathrm{ps}$ for a mass cut at $2.0 \mathrm{GeV} / c^{2}$ and $1.73 \pm 0.07$ and $1.54 \pm 0.10$ ps for a probability cut at 0.03 .) These have therefore not been quoted as systematic errors.

There was no evidence for any dependence upon the angles $\phi$ and $\theta$ of the B particle. The data have also been divided by the year in which it was recorded, and no year differs from the mean by more than one standard deviation.

To check the effect of the discrepancies seen in table 1 , the analysis of the simulation has been performed without the additional smearing of the reconstructed impact parameters. This increases the discrepancies at cuts 4) and 5) by a factor of 2, but hardly changes the lifetimes reconstructed from the simulation. This is evidence that these discrepancies are not important.

The data have also been fitted excluding those events where more than three tracks were rejected, 857 events. The results are $\left\langle\tau_{\text {charged }}\right\rangle=1.75 \pm 0.09$ and $\left\langle\tau_{\text {neutral }}\right\rangle=$ $1.59 \pm 0.12$. These too are consistent.

The results for the mean charged and neutral lifetimes are:

$$
\begin{aligned}
<\tau_{\text {charged }}> & =1.72 \pm 0.08(\text { stat }) \pm 0.06(\text { sys }) \mathrm{ps} \\
<\tau_{\text {neutral }}> & =1.58 \pm 0.11(\text { stat }) \pm 0.09(\text { sys }) \mathrm{ps} \\
\left\langle\tau_{\text {charged }}\right\rangle /\left\langle\tau_{\text {neutral }}>\right. & =1.09_{-0.10}^{+0.11}(\text { stat }) \pm 0.08(\text { sys })
\end{aligned}
$$

\subsection{Fit to B meson Lifetimes}

The data were also interpreted in terms of the lifetimes of the $\mathrm{B}^{0}$ and $\mathrm{B}^{+}$mesons, by assuming that the selected neutral $\mathrm{B}$ hadrons are composed of $\mathrm{B}^{0}, \mathrm{~B}_{s}^{0}$ and $\Lambda_{b}^{0}$, with the lifetimes of the $\mathrm{B}_{s}^{0}$ and $\Lambda_{b}^{0}$ as measured in independent analyses [14] and that all the charged $\mathrm{B}$ hadrons are $\mathrm{B}^{+}$. The $\mathrm{B}^{0}$ lifetime extracted in such a manner depended critically upon these assumptions, but the $\mathrm{B}^{+}$was relatively insensitive to them. The $\mathrm{B}_{s}^{0}$ and $\Lambda_{b}^{0}$ lifetimes were set to the current mean values [14] of $1.54 \pm 0.15 \mathrm{ps}$ and $1.16 \pm 0.11 \mathrm{ps}$ respectively and the $\mathrm{B}_{s}^{0}$ lifetime splitting was set to $\pm 9 \%[4]$. The fractions of $\mathrm{B}_{s}^{0}$ and $\Lambda_{b}^{0}$ at production were taken to be $12 \pm 4 \%$ and $8 \pm 4 \%$ respectively while the relative amount of $\mathrm{B}^{+}$was fitted. The results are shown in table 6 .

\begin{tabular}{|c|c|c|c|}
\hline Parameter & Data & \multicolumn{2}{|c|}{ Simulation } \\
\cline { 3 - 4 } & & Fit result & Correct value \\
\hline$\tau_{B^{+}}(\mathrm{ps})$ & $1.72 \pm 0.08$ & $1.56 \pm 0.05$ & 1.60 \\
$\tau_{B^{0}}(\mathrm{ps})$ & $1.63 \pm 0.14$ & $1.66 \pm 0.08$ & 1.60 \\
$\tau_{\text {confused }}(\mathrm{ps})$ & $0.40 \pm 0.39$ & $0.63 \pm 0.17$ & \\
$f_{0}$ & $0.43 \pm 0.05$ & $0.38 \pm 0.03$ & \\
$f_{\text {confused }}$ & $0.05 \pm 0.04$ & $0.06 \pm 0.02$ & \\
$\mathcal{P}_{1}$ & $0.20 \pm 0.13$ & $0.19 \pm 0.05$ & \\
$\mathcal{P}_{2}$ & $0.32 \pm 0.10$ & $0.33 \pm 0.05$ & \\
$\mathcal{P}_{3}$ & $0.60 \pm 0.10$ & $0.63 \pm 0.06$ & \\
\hline$\tau_{B^{+}} / \tau_{B^{0}}$ & $1.06_{-0.11}^{+0.13}$ & $0.94_{-0.06}^{+0.07}$ & 1.00 \\
$<\tau_{B}>(\mathrm{ps})$ & $1.64 \pm 0.06$ & $1.61 \pm 0.03$ & 1.60 \\
\hline
\end{tabular}

Table 6: Results for the $\mathrm{B}^{+}$and $\mathrm{B}^{0}$ lifetimes; only statistical errors are quoted. The $\mathrm{B}$ lifetimes were set to $1.6 \mathrm{ps}$ in the simulation for all states. 
Also shown in the table are the $\tau_{B^{+}} / \tau_{B^{0}}$ and $\left\langle\tau_{B}\right\rangle$. These values are a reparameterization of the result obtained for the $\mathrm{B}^{0}$ and $\mathrm{B}^{+}$lifetimes, but the errors have been evaluated separately. $\left\langle\tau_{B}\right\rangle$ is a measurement of the mean B lifetime using the relative abundances produced at LEP (see table 3 ) and assuming the values for the $\mathrm{B}_{s}^{0}$ and $\Lambda_{b}^{0}$ lifetimes mentioned above. As the measured lifetimes of the other B's were lower than the $\mathrm{B}^{+}$and $\mathrm{B}^{0}$ lifetimes the mean $\mathrm{B}$ lifetime was reduced.

The experimental systematic uncertainties have been taken from table 5, scaled by the increase in the statistical errors, as this reflects the increased change in the result to a given change in the inputs. The systematic error from the composition of the sample, estimated by changing the $\mathrm{B}_{s}^{0}$ and $\Lambda_{b}^{0}$ fractions as given in [12], varying their lifetimes as stated above, and altering the $\mathrm{B}_{s}^{0}$ lifetime splitting can be seen in table 7 . The mean $\mathrm{B}$ lifetime was relatively unaffected by these changes because the fitted $\mathrm{B}^{0}$ lifetime tends to compensate for them. Note that the mean B lifetime was exactly the same as that extracted assuming only two B species.

\begin{tabular}{|c|r|r|r|r|}
\hline Parameter & $\tau_{B^{+}}$ & $\tau_{B^{0}}$ & $\tau_{B^{+}} / \tau_{B^{0}}$ & $\left\langle\tau_{B}\right\rangle$ \\
\hline$f_{B_{s}}=0.08$ & +0.00 & -0.02 & +0.01 & -0.00 \\
$f_{B_{s}}=0.16$ & -0.00 & +0.02 & -0.01 & +0.00 \\
$\tau_{B_{s}}=1.39$ & -0.00 & +0.03 & -0.02 & -0.01 \\
$\tau_{B_{s}}=1.69$ & +0.00 & -0.05 & +0.04 & +0.00 \\
No $\tau_{B_{s}}$ splitting & -0.00 & +0.01 & -0.00 & +0.00 \\
$\pm 18 \% \tau_{B s}$ splitting & +0.00 & -0.02 & +0.01 & -0.01 \\
$f_{\Lambda_{b}}=0.06$ & +0.00 & -0.03 & +0.02 & +0.01 \\
$f_{\Lambda_{b}}=0.14$ & -0.00 & +0.03 & -0.02 & -0.01 \\
$\tau_{\Lambda_{b}}=1.05$ & +0.00 & +0.01 & +0.00 & -0.01 \\
$\tau_{\Lambda_{b}}=1.27$ & +0.00 & +0.00 & +0.00 & +0.01 \\
$\Xi_{b}^{-}$contribution & \pm 0.02 & +0.00 & +0.00 & +0.00 \\
\hline total & ${ }_{-0.02}^{+0.02}$ & ${ }_{-0.07}^{+0.05}$ & ${ }_{-0.05}^{+0.06}$ & ${ }_{-0.02}^{+0.01}$ \\
\hline
\end{tabular}

Table 7: The change in the measured lifetimes produced by making various assumptions about the minority $\mathrm{B}$ species.

The simulation predicts $0.2 \%$ of the $\mathrm{B}$ sample is $\Xi_{b}^{-}$. This is $0.4 \%$ of the charged B's, or around $1 \%$ when the $\Xi_{b}$ production is increased analogously to the $\Lambda_{b}^{0}$. DELPHI has measured the lifetime of the $\Xi_{b}[15]$. If we allow a lifetime range of zero to $3 \mathrm{ps}$, which is theoretically generous, it contributes a 0.02 ps error.

The final values for the lifetimes are:

$$
\begin{aligned}
\tau_{B^{+}} & =1.72 \pm 0.08(\text { stat }) \pm 0.06(\text { sys }) \pm 0.02(\text { composition }) \mathrm{ps} \\
\tau_{B^{0}} & =1.63 \pm 0.14(\text { stat }) \pm 0.11(\text { syst. })_{-0.07}^{+0.05}(\text { composition }) \mathrm{ps} \\
\tau_{B^{+}} / \tau_{B^{0}} & =1.06_{-0.11}^{+0.13}(\text { stat }) \pm 0.09(\text { sys })_{-0.05}^{+0.06}(\text { composition }) \\
\left\langle\tau_{B}\right\rangle & =1.64 \pm 0.06(\text { stat }) \pm 0.04(\text { sys })_{-0.02}^{+0.01}(\text { composition }) \mathrm{ps}
\end{aligned}
$$




\section{Summary}

From 1434425 hadronic $Z^{0}$ decays collected at the LEP collider with the DELPHI detector between 1991 and 1993, a sample of $1817 \mathrm{~B}$ hadron candidates with an estimated purity of $99 \%$ has been extracted. The results for the mean charged and neutral lifetimes are:

$$
\begin{aligned}
<\tau_{\text {charged }}> & =1.72 \pm 0.08(\text { stat }) \pm 0.06(\text { sys }) \text { ps } \\
\left\langle\tau_{\text {neutral }}>\right. & =1.58 \pm 0.11(\text { stat }) \pm 0.09(\text { sys }) \text { ps } \\
\left\langle\tau_{\text {charged }}\right\rangle /\left\langle\tau_{\text {neutral }}>\right. & =1.09_{-0.10}^{+0.11}(\text { stat }) \pm 0.08(\text { sys })
\end{aligned}
$$

The assumptions stated in the previous section allow the $\mathrm{B}^{+}$and $\mathrm{B}^{0}$ lifetimes to be measured. Combining the systematic uncertainties, these are as follows:

$$
\begin{aligned}
\tau_{B^{+}} & =1.72 \pm 0.08(\text { stat }) \pm 0.06(\text { sys }) \text { ps } \\
\tau_{B^{0}} & =1.63 \pm 0.14(\text { stat }) \pm 0.13(\text { syst. }) \mathrm{ps} \\
\tau_{B^{+}} / \tau_{B^{0}} & =1.06_{-0.11}^{+0.13}(\text { stat }) \pm 0.10(\text { sys }) \\
\left\langle\tau_{B}>\right. & =1.64 \pm 0.06(\text { stat }) \pm 0.04(\text { sys }) \text { ps }
\end{aligned}
$$

A composition systematic uncertainty was taken into account; it is significantly smaller for the $\mathrm{B}^{+}$than the $\mathrm{B}^{0}$ because the $\mathrm{B}^{+}$almost completely dominates the charged state.

These results are in agreement with, and supplant, our previous publication [1]. In an accompanying paper, DELPHI publishes a measurement of these lifetimes based upon D - lepton correlations [16], finding $\tau_{B^{+}}=1.61_{-0.16}^{+0.16} \pm 0.12 \mathrm{ps}$ and $\tau_{B^{0}}=1.61_{-0.13}^{+0.14} \pm 0.08 \mathrm{ps}$. The correlations between this analysis and the one presented here are very small, and the results have been combined to give:

$$
\tau_{B^{+}}=1.70 \pm 0.09 \mathrm{ps}
$$

and

$$
\tau_{B^{0}}=1.62 \pm 0.12 \mathrm{ps}
$$

The other measurements of these lifetimes presented to the 1994 Rochester Conference [14] by the LEP collaborations have been averaged using the methods of [12] and are: $\tau_{B^{+}}=1.57 \pm 0.11 \mathrm{ps}$ and $\tau_{B^{0}}=1.62 \pm 0.10 \mathrm{ps}$. These are in agreement with the measurements presented here.

\section{Acknowledgements}

We are greatly indebted to our technical collaborators and to the funding agencies for their support in building and operating the DELPHI detector, and to the members of the CERN-SL Division for the excellent performance of the LEP collider. 


\section{References}

[1] DELPHI Collaboration, P. Abreu et al., Phys. Lett. B 312 (1993) 253.

[2] NA14/2 Collaboration, Alvarez et al., Zeit. Phys. C 47 (1990) 539-546.

[3] H. Fritzsch and P. Minkowski, Phys Rep 73 (1981) 67.

[4] I. Bigi, B. Blok, M. Shifman, N. Uraltsev, A. Vainshtein in "B decays", $2^{\text {nd }}$ edition, Ed. S.Stone, World Scientific.

[5] DELPHI Collaboration, P. Aarnio et al., Nucl. Inst. and Meth. A303 (1991) 233.

[6] N. Bingefors et al., Nucl. Inst. and Meth. A328 (1993) 447.

[7] D. Johnson, D. Reid and W. Trischuk, "A Beamspot Database for Lifetime Measurements", DELPHI 92-36 PHYS 168, Geneva, 18 March 1992.

[8] JADE Collaboration, W. Bartel et al., Zeit. Phys. C 33 (1986) 23.

[9] T. Sjöstrand, Comp. Phys. Comm. 39 (1986) 347;

T. Sjöstrand and M Bengtsson, Comp. Phys. Comm. 43 (1987) 367.

[10] DELSIM Reference Manual, DELPHI 87-98 PROG 100, Geneva, July 1989.

[11] DELPHI Collaboration, P. Abreu at al., Zeit. Phys. C65 (1995) 555.

[12] LEP B lifetimes group. D. Bloch et al. "Averaging Lifetimes for B hadron species at LEP" ALEPH 94-198, DELPHI 94-164 PHYS 467, OPAL TN276.

[13] B. Franek, Rutherford Appleton Laboratory, RAL-85-026 (1985).

[14] P. Roudeau, proceedings of ICHEP XXVII, Glasgow, IOP, P.J. Bussey and I.G. Knowles eds, (1995) 325;

OPAL Collaboration, R. Akers et al., "Improved measurements of the $\mathrm{B}^{0}$ and $\mathrm{B}^{+}$ meson lifetimes", CERN-PPE/95-19, 1995.

[15] DELPHI Collaboration, P. Abreu et al., "Production of strange B-baryons decaying into $\Xi^{\mp}-\ell^{\mp}$ pairs at LEP", CERN-PPE/95-29, submitted to Zeit. Phys. C.

[16] DELPHI Collaboration, P. Abreu et al., “A Measurement of $\mathrm{B}^{+}$and $\mathrm{B}^{0}$ Lifetimes using D-lepton events in $Z^{0}$ Decays", to be submitted to Zeit. Phys. C. 